A hydrodynamic model for discontinuous shear-thickening in dense suspensions Mu Wang, Safa Jamali, and John F. Brady

Citation: Journal of Rheology 64, 379 (2020); doi: 10.1122/1.5134036

View online: https://doi.org/10.1122/1.5134036

View Table of Contents: https://sor.scitation.org/toc/jor/64/2

Published by the The Society of Rheology
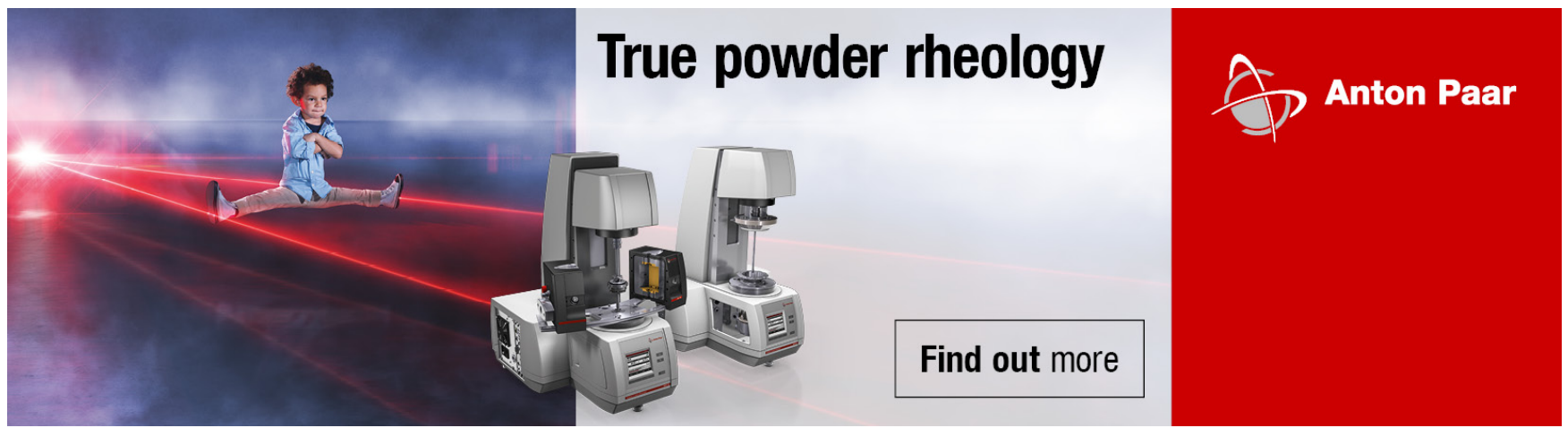


\title{
A hydrodynamic model for discontinuous shear-thickening in dense suspensions
}

\author{
Mu Wang, ${ }^{1, a)}$ Safa Jamali, ${ }^{2, b)}$ and John F. Brady ${ }^{3, c)}$ \\ ${ }^{1}$ Strategic Innovation \& Technology, The Procter \& Gamble Company, Cincinnati, Ohio 45217 \\ ${ }^{2}$ Department of Mechanical and Industrial Engineering, Northeastern University, Boston, Massachusetts 02115 \\ ${ }^{3}$ Division of Chemistry and Chemical Engineering, California Institute of Technology, Pasadena, California 91125
}

(Received 29 October 2019; final revision received 13 January 2020; published 5 March 2020)

\begin{abstract}
Restricted sliding or rotational motion of colloidal particles plays a key role in the emergence of discontinuous shear thickening (DST). From viscometric functions to the number of contacting neighbors under an applied deformation, a hindrance to sliding motion significantly changes the behavior of dense suspensions on all scales. In this work, implicitly by using a modified hydrodynamic model based on Stokesian dynamics and explicitly by solving for the hydrodynamics of nonsmooth colloids, we show that lubrication forces that arise from surface asperities effectively provide such constraints to tangential particle motion. A transition from continuous shear thickening to DST is observed as the surface roughness of the particles is systematically increased. In this hydrodynamic model for DST, normal stress differences remain negative in the shear-thickened state (STS). Study of the spatial stress distribution indicates the onset of DST to be a highly localized event; however, particle self-diffusivity and the microstructural network suggest a rather uniform structure in the STS. (C) 2020 The Society of Rheology. https://doi.org/10.1122/1.5134036
\end{abstract}

\section{INTRODUCTION}

Dense suspensions of colloidal particles show rich and complex rheological behaviors under an applied deformation. At low deformation rates, the viscosity of the suspension decreases as the rate of deformation increases-the suspension shear-thins. However, upon reaching a critical stress, the suspension begins to show resistance to large deformations and the viscosity increases with increasing deformation rate - shear thickening (ST) [1-4]. The suspension viscosity in the ST regime can grow either gradually with deformation rate, referred to as continuous shear-thickening (CST), or abruptly, with a jump in viscosity upon small increase in the applied deformation rate, commonly referred to as discontinuous shear-thickening (DST). In early studies of ST behavior, the term dilatancy was commonly used to describe the ST behavior [5-8]; however, dilatancy refers to the volume expansion of the particulate matter (also commonly observed in granular materials) and does not require a viscosity increase.

While studies of the viscosity changes of dense suspensions under flow date back to 1958 [8], it was not until 1994 that Laun [9] showed distinct signatures in normal stress differences under flow. These measurements showed both normal stress differences, $N_{1}$ and $N_{2}$, to be negative and large in magnitude for ST suspensions. Aral and Kalyon [10] found an increasing $\left|N_{1}\right|$ as the volume fraction of the particle

Note: This paper is part of the special issue on Physics of Dense Suspensions.

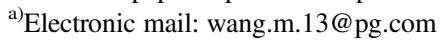

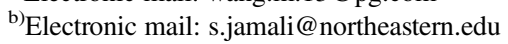

${ }^{c)}$ Author to whom correspondence should be addressed; electronic mail: jfbrady@caltech.edu

(C) 2020 by The Society of Rheology, Inc.

J. Rheol. 64(2), 379-394 March/April (2020) phase increases, and Lee et al. [11] also reported negative first normal stress differences. Accurate measurement of normal stress differences in Silica particles by Cwalina and Wagner [12] showed both normal stress differences to be large and negative in the ST regime. These measurements stand in contrast to several other studies where the first normal stress differences, $N_{1}$, are reported to be large and positive in the ST regime [13-17]. Regardless of the differences in the reported sign of $N_{1}$, it is clear that all material functions of dense suspensions under flow exhibit explicit rheological transitions in the ST regime.

To describe the underpinning of such extraordinary rheological behavior, theories and computational models have been developed over the past three decades. The hydrodynamic model, enabled by the development of the Stokesian dynamics (SD) computational platform [18,19], predicts an increase in "hydrodynamic viscosity" (that is the contribution of hydrodynamic stresses between interacting colloidal particles to the shear viscosity) at large deformation rates, which dominates the decreasing Brownian contribution, and results in overall ST behavior. As the applied deformation rate is increased, the gap between two nearby particles is progressively getting smaller [20-22], and since the lubrication stresses scale inversely with the separation distance between the particles, $1 / h$, these dissipative hydrodynamic forces become significantly larger at high deformation rates. As a result, particles are locked together giving rise to large lubrication-connected aggregates called "hydroclusters" that resist flow. In these simulations, only hydrodynamic shear and Brownian forces are present, and so the rheological behavior is described by a simple dimensionless group (in addition to volume fraction), the Péclet number, $\mathrm{Pe}=6 \pi \eta_{0} \dot{\gamma} a^{2} /\left(k_{\mathrm{B}} T / a\right)$, which represents the ratio of shear forces to thermal forces acting on a particle. Here, $a$ is the 
radius of a particle, $\eta_{0}$ is the viscosity of background suspending fluid, and $\dot{\gamma}$ is the imposed deformation rate. The microstructures described by the pair-correlation function of suspensions under shear flow in SD simulations exhibited a fore-aft asymmetry with accumulation in the compressional axis and depletion in the extensional axis. This microstructural feature can be used to describe the sign of normal stress difference in ST suspensions [23]. Careful scattering and flow reversal experiments on dense suspensions [24,25] confirmed the role of hydrodynamic stresses and observed microstructures in agreement with those predicted by SD simulations. Examination of the relative tangential motion of a particle pair in the lubrication regime shows that the resistance to tangential motion, i.e., the nonsqueezing mode of lubrication, behaves as $\log (1 / h)$ and hence is much weaker than the perpendicular squeezing mode [20-22,26]. This weak logarithmic resistance to tangential motion for smooth spherical particles means that particles can easily escape a hydrocluster resulting in a gradual or CST behavior.

Utilizing the squeezing motion in other techniques such as dissipative particle dynamics (DPD) has enabled simulations of bimodal suspensions [27] and soft particles [28], which exhibit strong CST behaviors with negative normal stress differences. Nonetheless, as with SD, simulations with only squeezing lubrication do not predict DST. In all simulations performed using squeezing mode lubrication, CST behavior with a three- to sixfold increase in the viscosity in the ST regime has been obtained, with no clear indication of a viscosity plateau in the shear-thickened state (STS). It should also be noted that based on lubrication hydrodynamics, the singularity at contact in the hydrodynamic forces precludes any direct contact between smooth particles. Most real particles are not smooth, and as we shall see, small scale surface asperities can change the hydrodynamic flows and break the lubrication layer [29]. This observation inspired the development of a frictional contact model for viscous suspensions similar to that for dry granular materials.

In the frictional contact model, the relative tangential motions are governed by a stick-slide scenario based on Coulomb's law of friction that is proposed to result from the breakage of the fluid lubrication layer separating two colloidal particles [30-33]. In a simulation, the numerical limitation of a finite time step results in particles overlapping. One can presume that in such overlap situations, the hydrodynamics have broken down and solid contacts dominate. With the inclusion of tangential forces from friction, DST is observed followed by a plateau in the STS. This perspective is in line with the theoretical approach proposed by Wyart and Cates [34], where two maximum packing fractions are envisioned for frictional and frictionless particles. Experiments on rough particles [13,35-38] also indicate that a constraint to sliding/ rolling motion of particles is essential to recover the DST behavior. Due to the tangential nature of frictional forces, and the lack of competing forces in the ST regime, frictional contact models consistently predict positive and large $N_{1}$ values.

These recent developments and experimental observations have led to an emergence of consensus that a constraint to rolling/sliding motion of particles is key to understand dense suspension flow behaviors. We recently showed that by careful and detailed consideration of the role of surface asperities [39], the full hydrodynamic model reproduces not only the DST behavior but can also capture a negative $N_{1}$ in the STS. In this paper, we present two different models for incorporating surface asperities: an implicit model with modified lubrication interactions to account for surface asperities, and an explicit model that constructs surface roughness by building composite particles. We show that both models reproduce similar viscometric functions under flow. In this work, we focus on how hydrodynamics and particle surface asperities contribute to the onset of a viscosity jump in the DST. The recent experimental and numerical observations on the sign inversion of $N_{1}$ at higher imposed flow, currently understood as the transition between the hydrodynamic dominant and friction dominant rheology behaviors [13,16,36], are beyond the scope of the present work.

\section{METHODS}

\section{A. Motivation}

Our model is motivated by the fact that the microscopic origin of suspension stresses cannot be determined from macroscopic stress measurements alone. Additional microscopic probes and analysis on suspension mechanics, microstructures, and stress states are necessary to pinpoint the microscopic origin.

This is demonstrated by a simple computational experiment: consider a string of rigid, smooth, neutrally buoyant, and non-Brownian particles of radii $a$ in a Newtonian solvent of density $\rho_{0}$ and viscosity $\eta_{0}$ arranged in a straight line along the compressional axis in a simple shear flow of strain rate $\dot{\gamma}$ as is shown in Fig. 1(b). Given the static geometric configuration, we consider different interaction scenarios among particles: (a) the particles interact only through hydrodynamics, i.e., no other forces are imposed on the particles, $F^{\mathrm{ext}}=0$. (b) In addition to hydrodynamic interactions (HIs), the particles are subject to a short-range (SR),

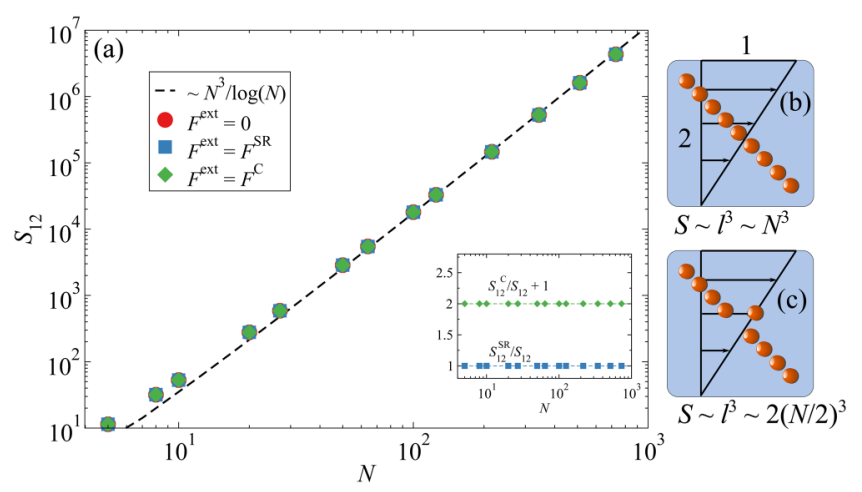

FIG. 1. The velocity-velocity gradient direction total stress $S_{12}$, scaled with $\eta_{0} \dot{\gamma} a^{3}$, as a function of the particle number $N$ for a string of $N$ particles in a simple shear flow. The string is aligned with the compression axis of the flow as indicated in the inset. The gap spacing between particles is $r / a-2=10^{-8}$. The particles in the string are interacting: (a) without any external forces, $F^{\text {ext }}=0$ (red circles); (b) with short-range repulsive force, $F^{\mathrm{ext}}=F^{\mathrm{SR}}$; and (c) with a constraint force for the particle string to move as a rigid body, $F^{\text {ext }}=F^{\mathrm{C}}$. The inset shows the ratio of the total stress in (b) and (c) relative to (a). The results for (c) are shifted for clarity. 
pairwise repulsive force $F^{\mathrm{SR}}$. The force on particles $\alpha$ due to particle $\beta, \mathbf{F}_{\alpha \beta}$, is

$$
\mathbf{F}_{\alpha \beta}=F_{0} \exp (-\tau h) \mathbf{e}_{\alpha \beta},
$$

where $\mathbf{e}_{\alpha \beta}$ is the unit vector connecting the centers of the two particles pointing toward particle $\alpha$. For illustration, we set the force magnitude $F_{0}=100 \eta_{0} \dot{\gamma} a^{2}$ and $\tau a=100$. This case is designated as $F^{\mathrm{ext}}=F^{\mathrm{SR}}$. (c) On top of HIs in case (a), the particles are subject to constraint forces $F^{\mathrm{C}}$ to ensure the string moves as a rigid body. We compute the total stress of the string $S_{12}$ for different particle numbers $N$, corresponding to different lengths $\ell \sim a N$.

For particles that are small enough such that the Reynolds number $\operatorname{Re}=\rho_{0} a^{2} \dot{\gamma} / \eta_{0} \ll 1$, the solvent velocity field $\mathbf{u}(\mathbf{r})$ and pressure field $p(\mathbf{r})$ are governed by the Stokes equation, i.e.,

$$
\eta_{0} \nabla^{2} \mathbf{u}=\nabla p, \nabla \cdot \mathbf{u}=0
$$

The linearity of Eq. (2) implies that the force, torque, and stress are linearly proportional to the particle velocity, angular velocity, and imposed strain rate. The standard resistance formulation for particles in the Stokes flow [40,41] is

$$
\left[\begin{array}{l}
\mathbf{F}^{E} \\
\mathbf{S}^{E}
\end{array}\right]=-\left[\begin{array}{ll}
\mathbf{R}_{F U} & \mathbf{R}_{S U} \\
\mathbf{R}_{F E} & \mathbf{R}_{S E}
\end{array}\right] \cdot\left[\begin{array}{c}
\mathbf{U}-\mathbf{U}^{\infty} \\
-\mathbf{E}^{\infty}
\end{array}\right],
$$

where, for instance, $\mathbf{R}_{F U}$ is the resistance function connecting the particle hydrodynamic force/torque and the particle translational/rotational velocity, $\mathbf{F}^{E}$ is the generalized force/torque on the particles due to flow, $\mathbf{S}^{E}$ is the flow contribution to stresslet, $\mathbf{U}$ are the generalized particle velocities including the linear and angular velocities, and $\mathbf{U}^{\infty}$ and $\mathbf{E}^{\infty}$ are imposed flow and strain rate, respectively. Solving Eq. (3), the stresslet is

$$
\mathbf{S}^{E}=\left(-\mathbf{R}_{S U} \cdot \mathbf{R}_{F U}^{-1} \cdot \mathbf{R}_{F E}+\mathbf{R}_{F E}\right) \cdot \mathbf{E}^{\infty}
$$

and the total stress for case (a) can be straightforwardly computed. For cases (b) and (c), where there is an external force $\mathbf{F}^{\mathrm{X}}$ with $\mathrm{X}$ designating its origin, the stress contribution now becomes

$$
\mathbf{S}^{\mathrm{X}}=-\mathbf{R}_{S U} \cdot \mathbf{R}_{F U}^{-1} \cdot \mathbf{F}^{\mathrm{X}}-\mathbf{x} \mathbf{F}^{\mathrm{X}},
$$

where $\mathbf{x}$ is the particle position. For case (c), the constraining force $\mathbf{F}^{\mathrm{C}}$ that ensures the particle assembly moves as a rigid body is evaluated based on the framework of [42] with adjustments on the imposed shear flow. The total stress of the string is the sum of stress contributions from all particles.

Figure 1(a) shows the total shear stress of the particle string, $S_{12}$, as a function of the string particle number $N$, with different interacting scenarios outlined above. The particles are nearly touching each other, with the gap spacing $h=r / a-2=10^{-8}$, typical for strongly sheared suspensions. Despite distinct particle interactions, $S_{12}$, the three cases are virtually identical to each other for all $N$ considered. This is better illustrated in the inset of Fig. 1(a), which shows the ratio of the shear stresses with interacting forces to the purely hydrodynamic one. Regardless of the underlying mechanisms that control particle interactions, the total shear stress of the string is the same. Therefore, one cannot tell from macroscopic suspension stresses the underlying particle interactions, or if and how interparticle forces affect the stress of nearly touching particle strings.

From hydrodynamics, and as is shown in Fig. 1(a), the stress of a string of length $\ell=a N$ scales as $\sim \ell^{3}$, and breaking the string into two reduces the total stress of the $N$ particle system to $2(\ell / 2)^{3}=\ell^{3} / 4$. If the string is broken into $M$ pieces, the total stress is reduced to $\ell^{3} / M^{2}$. If the suspension stress is dominated by the largest stress-sustaining cluster, the stress reduction by string breakage could explain the orders of magnitude lower shear viscosity in CST compared to DST. The easily broken cluster in CST is due to the tangential sliding/rotation motion particles, where the hydrodynamic forces only scale as $\log \left(h^{-1}\right)$. Consequently, a particle can easily slip out sideways breaking the cluster into two. In Brownian colloidal suspensions, a particle in a stresssustaining string or cluster could easily wander off due to thermal fluctuations, leading to drastically reduced viscosity. The breakage of strings leading to significant stress reduction is further illustrated in Fig. 1(c). Therefore, the role of friction is not to bear the load but to restrict particle tangential movement to prevent string breakage in frictional systems.

The computational experiment in Fig. 1 suggests that there may be multiple plausible mechanisms including frictional contact forces that lead to the experimentally observed viscosity jump in DST. The key is to restrict sliding (or rolling) tangential motion. We propose two possible hydrodynamic mechanisms based on particle surface asperities in Secs. II B and II C.

\section{B. Modified hydrodynamic interaction model}

We motivate our model by considering the hydrodynamic interactions between two particles with radii $a$ sliding parallel to each other at velocity $U$ as is shown in Fig. 2. When the particle gap spacing is small, i.e., $h \ll a$, lubrication theory [29] shows that the tangential hydrodynamic forces between the two particles diverge as $\log \left(h^{-1}\right)$. This is a much weaker divergence compared to the case when the particles are moving toward each other, where the hydrodynamic force diverges as $h^{-1}$. However, as is illustrated in Fig. 2, when the particle surfaces are not smooth, the surface asperities may alter the sliding motion hydrodynamic interactions in two ways: (a) it quantitatively reduces the particle gap size to $h^{\prime}<h$, and (b) more importantly, it introduces local compressive squeezing motion to the purely sliding movement as the two rough surfaces approach each other. These compressive motions qualitatively alter the divergence of hydrodynamic interactions from $\log \left(h^{-1}\right)$ to $h^{\prime-1}$, as shown at the bottom right panel of Fig. 2. These stronger hydrodynamic interactions may produce tightly locked particle pairs under shear, leading to increased viscosities.

Hydrodynamic interactions are different from frictional resistance forces in several ways: (a) their natural scales are different: hydrodynamic interactions scale linearly with the imposed flow, i.e., $\propto \dot{\gamma}$, while interparticle forces scale with 


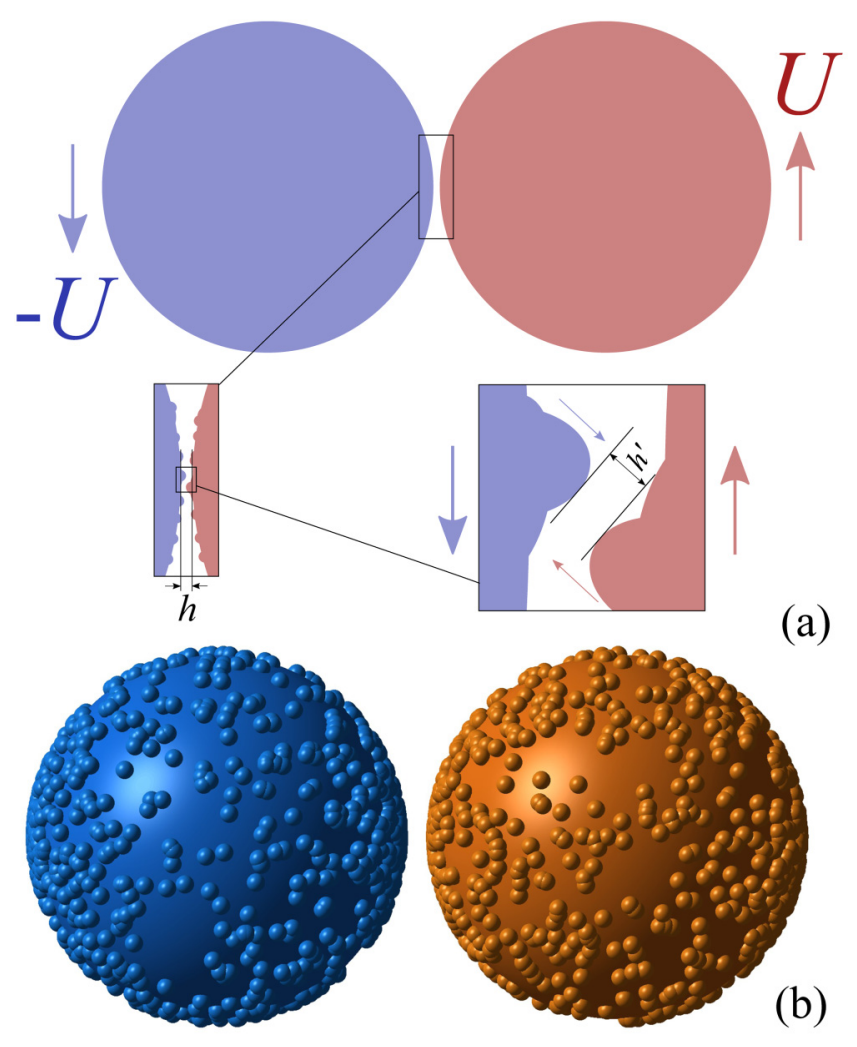

FIG. 2. (a) Illustration of how particle surface asperities affect hydrodynamic interactions. For two particles in tangential motion (either sliding or rotating) with gap spacing $h$ and velocity $U$, the hydrodyanmic forces/ torques for smooth particles diverge as $\sim \log \left(h^{-1}\right)$. Surface asperities create compressive movement between particles with spacing $h^{\prime}$, leading to strong hydrodynamic forces/torques that diverge as $\sim 1 / h^{\prime}$. (b) Snapshot of two rough particles in DPD computations.

the underlying intermolecular interactions, that is, $\propto k T$. (b) Hydrodynamic interactions are dissipative and resist particles' relative tangential/sliding movements, not just normal motions. Therefore, they prevent particles from approaching to, as well as departing from, each other. On the other hand, frictional contact forces generally only prevent particles from approaching each other. This distinction may explain the positive first normal stress difference $N_{1}$ observed in frictional based simulations [30], as frictional forces only push the particles apart.

A simple way to capture hydrodynamic interactions of rough particles as illustrated in Fig. 2 is to strengthen the weak, logarithmically diverging hydrodynamic interactions with a polynomial one. Assuming the height of the surface roughness is characterized by a length scale $h_{0}$, and the surface roughness leads to a stronger inverse gap spacing divergence $\sim \alpha / h$, where $\alpha$ is the strength of the divergence, we add the following expression to the weakly diverging lubrication expressions when the gap spacing $h<h_{0}$,

$$
f(h)=f\left(h ; \alpha, h_{0}\right)=\frac{\alpha}{h}+\frac{2 \alpha}{h_{0}^{3}} h^{2}-\frac{3 \alpha}{h_{0}^{2}} h .
$$

For two particles of size $a_{1}$ and $a_{2}$ with center-to-center distance $r$, the gap spacing is $h=r-a_{1}-a_{2}$. Equation (6) emphasizes that $h$ is the variable and $\alpha$ and $h_{0}$ are adjustable
TABLE I. The sign of $f\left(h ; \alpha, h_{0}\right)$ in Eq. (6) with respect to different components of the resistance functions.

\begin{tabular}{lcccc}
\hline \hline$R$ & $Y^{A}$ & $Y^{G}$ & $Y^{M}$ & $Z^{M}$ \\
\hline 11 & + & + & + & + \\
12 & - & - & + & + \\
21 & - & + & + & + \\
22 & + & - & + & + \\
\hline \hline
\end{tabular}

parameters and ensures that the strong inverse gap spacing divergence is added smoothly from the cutoff distance $h_{0}$, i.e., $f\left(h_{0}\right)=f^{\prime}\left(h_{0}\right)=0$. The length $h_{0}$ sets the height or range of the asperities and the magnitude $\alpha$ measures the amount of surface roughness. The form of Eq. (6) ensures that the Brownian displacement, specifically, $k T \nabla \cdot \mathbf{D}$ with $\mathbf{D}$ the diffusion tensor, is continuous.

Despite its simplicity, incorporating Eq. (6) into computational frameworks such as Stokesian dynamics requires some considerations. First, the sign of $f\left(h ; \alpha, h_{0}\right)$ added to the hydrodynamic resistance functions must be physically consistent. Second, while seven resistance functions diverge weaker than $\sim h^{-1}$, i.e., following the notation of Kim and Karilla [29], $Y^{A}, Y^{B}, X^{C}, Y^{C}, Y^{G}, Y^{H}, Y^{M}, Z^{M}$, only the ones that have a $\sim h^{-1}$ divergence counterpart need modifications. For instance, we modify $Y^{A}$ with $f(h)$ because $X^{A} \propto h^{-1}$ as $h \rightarrow 0$. Incorporating the divergence of Eq. (6) to all seven aforementioned resistance functions could destroy the positive definiteness of the grand resistance tensor, leading to unphysical rheological behaviors or even numerical convergence failure. Finally, while each resistance function could have a distinct divergence magnitude $\alpha$, for simplicity we assume that the same $\alpha$ is appropriate for all modified resistance functions.

Table I summarizes the resistance functions modified by Eq. (6) and the associated signs in their pairwise component. Other than these adjustments, other mechanisms in the SD computational framework are unchanged [43].

\section{Explicit rough particle model}

As an alternative to modified hydrodynamic interaction presented above, one can explicitly model the surface asperities through the inclusion of smaller spherical particles, referred to as patches here, onto the surface of the base particle. This is done through a modified DPD scheme [27,28], for rough composite colloids interacting hydrodynamically.

\section{RESULTS AND DISCUSSIONS}

We consider a neutrally buoyant bidisperse (to prevent shear-induced crystallization) Brownian suspension with particle size ratio $\lambda=a_{l} / a_{s}=1.4$ where $a_{l}$ and $a_{s}=a$ are the radii of the larger and the smaller particles, respectively. The particle volume ratio is $y_{s}=0.5$, where $y_{s}$ is the fraction of solid volume occupied by smaller particles. A simple shear flow with strain rate $\dot{\gamma}$ is imposed at a fixed total solid volume fraction $\phi$. The ratio of the shear to Brownian force defines the Péclet number, $\mathrm{Pe}=\dot{\gamma} a^{2} / d_{0}$, where $d_{0}$ is the Stokes-Einstein diffusivity $d_{0}=6 \pi \eta_{0} a /(k T)$ of smaller 
particles and $\eta_{0}$ is the solvent viscosity. We assume the particles are small enough that the hydrodynamic interactions are described by Eq. (3). In addition to hydrodynamic interactions, the particles are also subject to short-range interparticle forces in Eq. (1).

\section{A. Rheology of rough particle suspensions}

\section{Influence of hydrodynamic thickening parameters}

We compute the rheology of dense colloidal suspensions with rough surfaces using SD [40] with modified lubrication interactions as described in Sec. II B with a modest number of particles $N=30$ and using the explicit DPD simulations. For SD simulations, each run lasts at least 100 time units with a step size of 0.001 . When $\mathrm{Pe} \geq 1$, the simulation time is scaled as $1 / \dot{\gamma}$, and when $\mathrm{Pe}<1$, the simulation time is scaled with $a^{2} / d_{0}$. Unless otherwise stated, the particles interact purely through hydrodynamic interactions.

The influence of parameters $\alpha$ and $h_{0}$ in Eq. (6) on the suspension shear viscosity $\eta_{s} / \eta_{0}$ is shown in Fig. 3 for bidisperse suspensions at $\phi=0.5$. The solid lines are results for smooth particles. Figure 3(a) shows that $\alpha$ plays a dominant role in altering the suspension shear viscosity. As $\alpha$ increases, the shear viscosity at high Pe grows proportionally to $\alpha$, making it the dominant contributing factor to the observed shear thickening. The roughness characteristic size
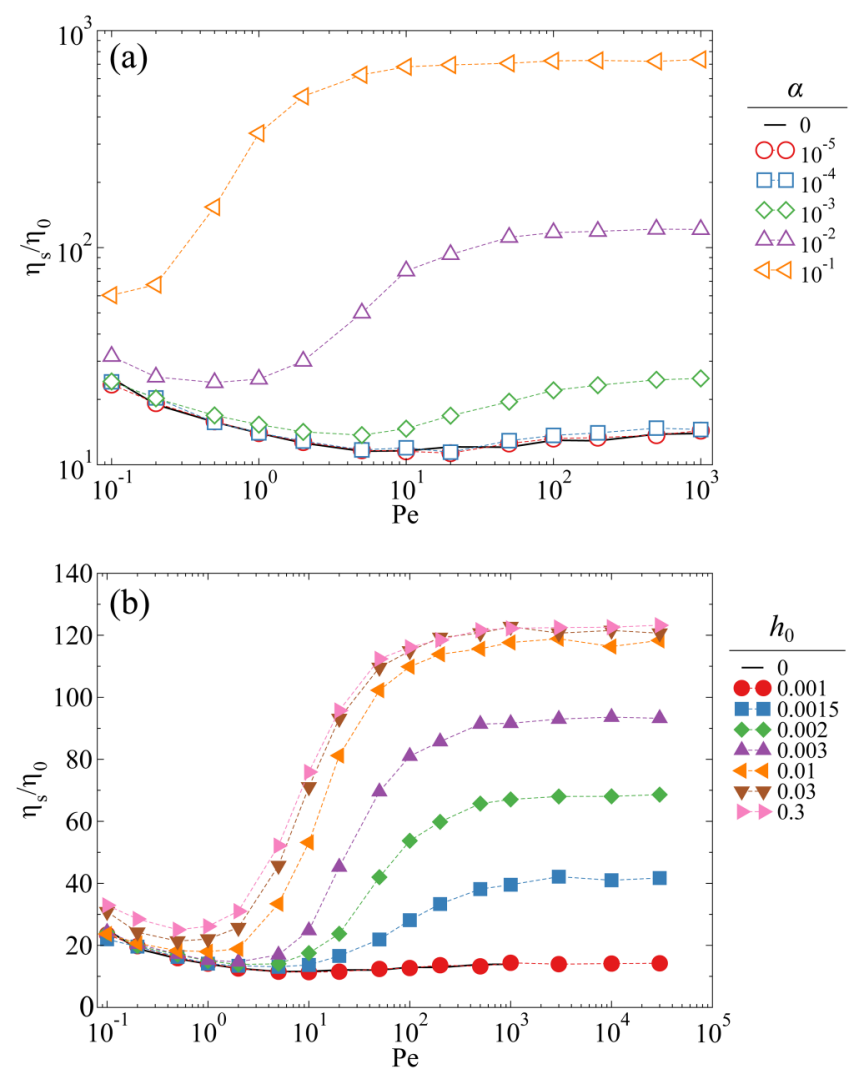

FIG. 3. (a) Effects of the asperity magnitude $\alpha$ on the suspension shear viscosity $\eta_{s} / \eta_{0}$ as a function of Péclet number Pe with $h_{0}=0.1$, (b) effects of the cutoff distance $h_{0}$ scaled with $\left(a_{i}+a_{j}\right)$ on the suspension shear viscosity $\eta_{s} / \eta_{0}$ as a function of Pe with $\alpha=0.01$. The suspension is bidisperse with size ratio $\lambda=1.4$ and volume ratio $y_{s}=0.5$ at a total volume fraction $\phi=0.5$. The solid lines in (a) and (b) are results for smooth particles. $h_{0}$ plays a similar role in increasing the shear viscosity $\eta_{s} / \eta_{0}$, as is illustrated in Fig. 3(b). As $h_{0}$ increases, $\eta_{s} / \eta_{0}$ increases with Pe and collapses to a limiting behavior for $h_{0}>0.01$.

Figure 4 shows the effects of volume fraction $\phi$ for rough bidisperse suspensions with $\alpha=0.01$ and $h_{0}=0.003$ from $\phi=0.45$ to 0.58 . As expected, increasing volume fraction increases the suspension shear viscosity for all Pe. Figure 4(a) also illustrates that the shear thinning behavior at low $\mathrm{Pe}$ is more prominent as the volume fraction $\phi$ increases. For suspensions with $\phi \geq 0.54$, a minimum shear viscosity emerges at moderate Pe. Figure 4(b) displays the suspension shear viscosity using the stress Péclet number $\mathrm{Pe}_{\sigma}=6 \pi a^{3} \sigma /(k T)=\left(\eta_{s} / \eta_{0}\right) \mathrm{Pe}$. With the stress Péclet number, the suspension shear thickening at different volume fractions $\phi$ takes place at roughly the same onset stress, noted as $\sigma_{\text {on }}$; here, the onset stress Péclet number $\sigma_{\text {on }} \approx 600$.

The influences of Pe and $\phi$ on the first and second normal stress differences, $N_{1}$ and $N_{2}$, respectively, are illustrated in Figs. 5(a) and 5(b) for the same suspensions as in Fig. 4. In contrast to simulations with frictional forces, Fig. 5(a) shows that the first normal stress difference $N_{1}$ remains negative at large Péclet numbers even with hydrodynamic interactions modified by $f\left(h ; \alpha, h_{0}\right)$. Physically, the first normal stress becomes negative because hydrodynamic interactions are necessary to pull particles apart. This mechanism is absent in frictional models. The normal stress differences are scaled with the strain rate $\dot{\gamma}$ and approach a constant value at higher Pe. Furthermore, the first normal stress difference $N_{1}$ with $\phi \geq 0.45$ exhibits limited volume fraction variation. However, $N_{2}$ is always negative, and compared to $N_{1}$, it is more sensitive to $\phi$.

\section{Effects of short-range repulsive forces}

Short-range repulsive forces also play a crucial role in suspension rheology. We introduced a simple short-range repulsive force in Eq. (1). Note that contrary to Sec. II A, here we adopt a thermal scale for the force magnitude $F_{0}$, i.e., $F_{0} \sim k T / a$. Figure 6(a) shows the effects of repulsive force magnitude $F_{0}$, scaled with $k T / a$, with fixed $\tau a=100$, on the suspension shear viscosity $\eta_{s} / \eta_{0}$. The short-range forces push particles apart, delaying the onset of shear thickening to a higher shear rate. Expressing suspension rheology using the stress Péclet number $\mathrm{Pe}_{\sigma}$ shows that the short-range repulsive force also shifts the shear thickening onset stress. Rescaling the shear viscosity with the onset stress $\sigma_{\text {on }}$, as illustrated in Fig. 6(b), collapses the flow curves with different $F_{0}$ values, suggesting that the fundamental aspects of shear thickening remain unchanged. The critical stress for shear thickening scales as $a^{-2}$ for nonhydrodynamic interparticle forces, whereas it scales as $a^{-3}$ for purely hydrodynamic Brownian suspensions

\section{B. Comparison with the experiments of Cwalina and Wagner}

\section{Suspension bulk rheology}

We test whether the modified hydrodynamic interactions described in Sec. II B could capture the experimentally 


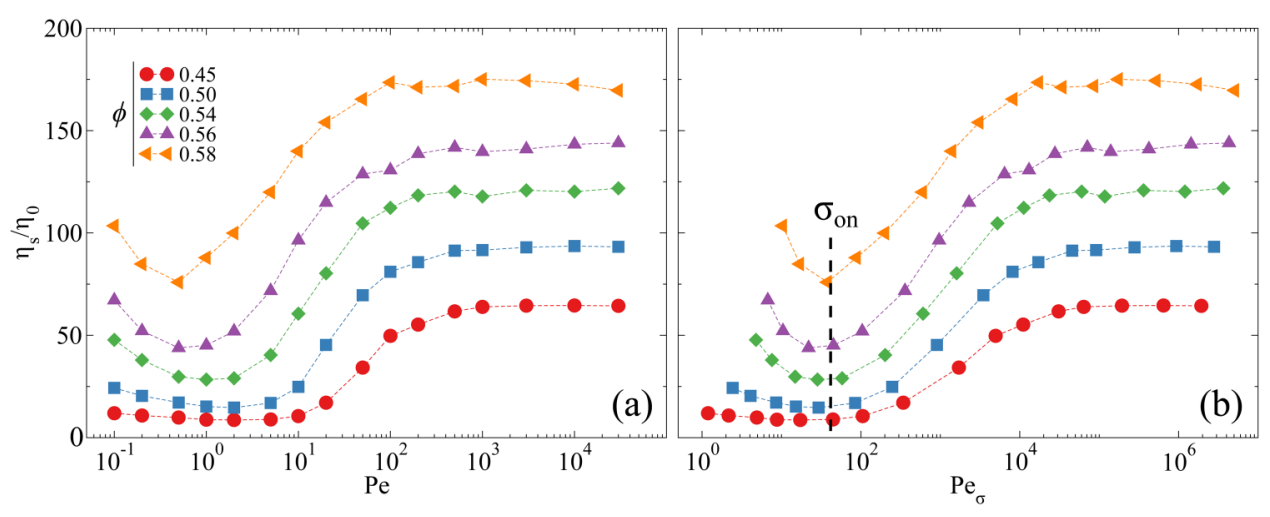

FIG. 4. Influence of the volume fraction $\phi$ on the suspension shear viscosity $\eta_{s} / \eta_{0}$ for suspensions with asperity magnitude $\alpha=0.01$ and $h_{0}=0.003$. (a) The shear viscosity as a function of the shear rate Péclet number Pe; (b) the shear viscosity as a function of the stress Péclet number Pe $e_{\sigma}=\left(\eta_{s} / \eta_{0}\right)$ Pe. The onset stress of shear thickening is identified as $\sigma_{\text {on }}$.

observed bulk rheology measurements in Cwalina and Wagner [12] at $\phi=0.48,0.50$, and 0.52. To make the comparison, we adjust the volume fraction $\phi$, the shear thickening parameters $\alpha$ and $h_{0}$, and the short-range repulsive force parameters $F_{0}$ and $\tau$ in the computations and seek the set of parameters that best represents the experimental shear viscosity measurements $\eta_{s} / \eta_{0}$. For simplicity, we fixed the short-range force inverse interaction distance $\tau a=100$ and the hydrodynamic cutoff distance $h_{0} / a=0.0015$. The parameter search is completed manually through trial and error using SD simulations with $N=30$ in bidisperse suspensions. Other optimization strategies could improve the parameter search efficiency. The resulting parameters are shown in Table II. The higher than experimentally fitted volume fractions $\phi$ are likely because in simulations the suspensions are bidisperse but in experiments the suspensions are polydisperse.

During the fitting process, we fix the set of parameters for each series of simulations and compare the computed suspension shear viscosity $\eta_{s} / \eta_{0}$ with the experimental results at a fixed volume fraction. This is different from the approach of [31], where the parameter adjustments are performed at every data point. After the optimal sets of parameters are found from the SD simulations, we ran another set of simulations with the same parameters in Table II using a near-field version of the Spectral Ewald Accelerated Stokesian Dynamics [43] (SEASD-nf) with $N=500$ to investigate the suspension structural features.

Figure 7 shows a good agreement between the experimental data and the simulation results for the suspension shear viscosity $\eta_{s} / \eta_{0}$ as a function of the Péclet number Pe. The experimental data are shown in solid lines, and the SEASD-nf simulations are shown in symbols. Simulations with the modified hydrodynamic interactions in Sec. II B not only captured the mild continuous shear thickening in experiments at $\phi=0.48$ but also the dramatic discontinuous shear thickening in experiments at $\phi=0.52$. The fitting
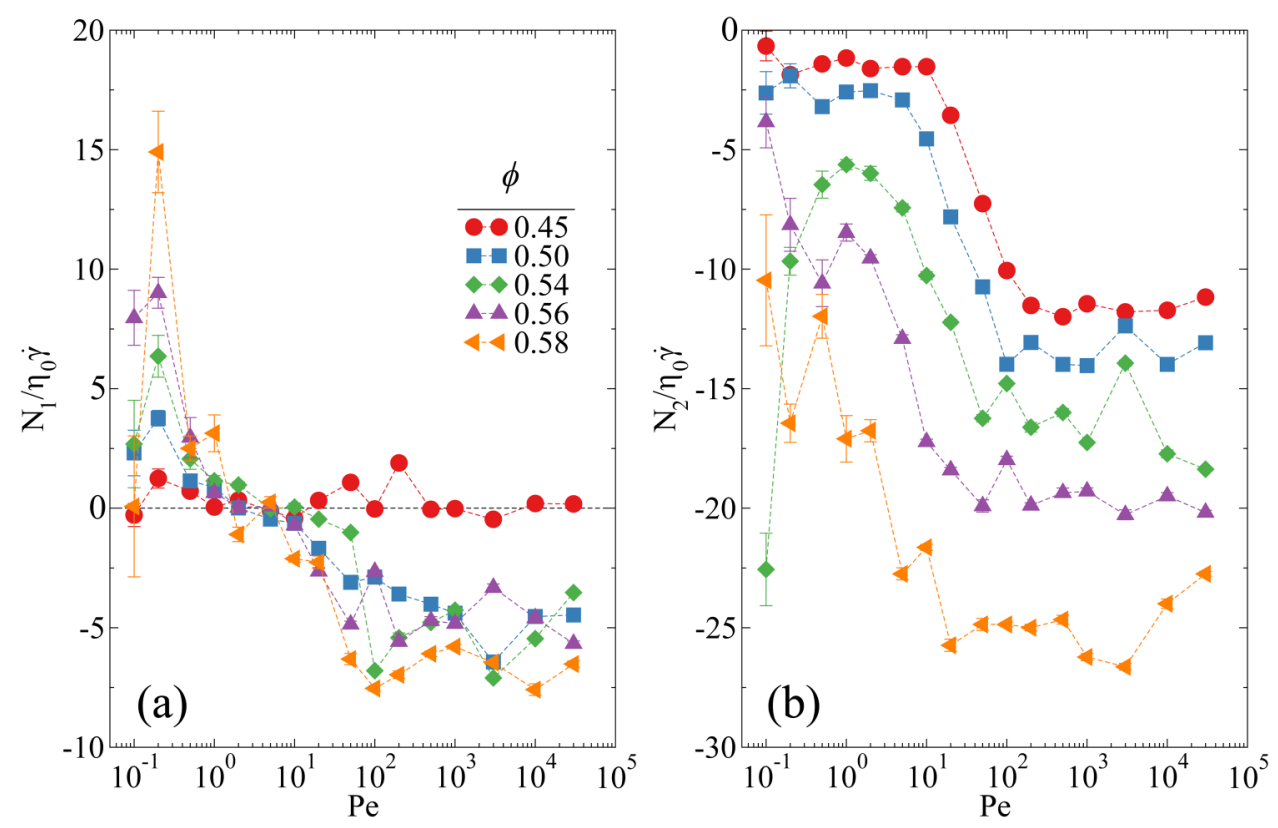

FIG. 5. Influence of volume fraction $\phi$ on the suspension first and second normal stress differences, $N_{1}$ and $N_{2}$, for suspensions with $\alpha=0.01$ and $h_{0}=0.003$ : (a) the scaled first normal stress different $N_{1} /\left(\eta_{0} \dot{\gamma}\right)$ and (b) the scaled second normal stress different $N_{2} /\left(\eta_{0} \dot{\gamma}\right)$ as functions of Pe. 


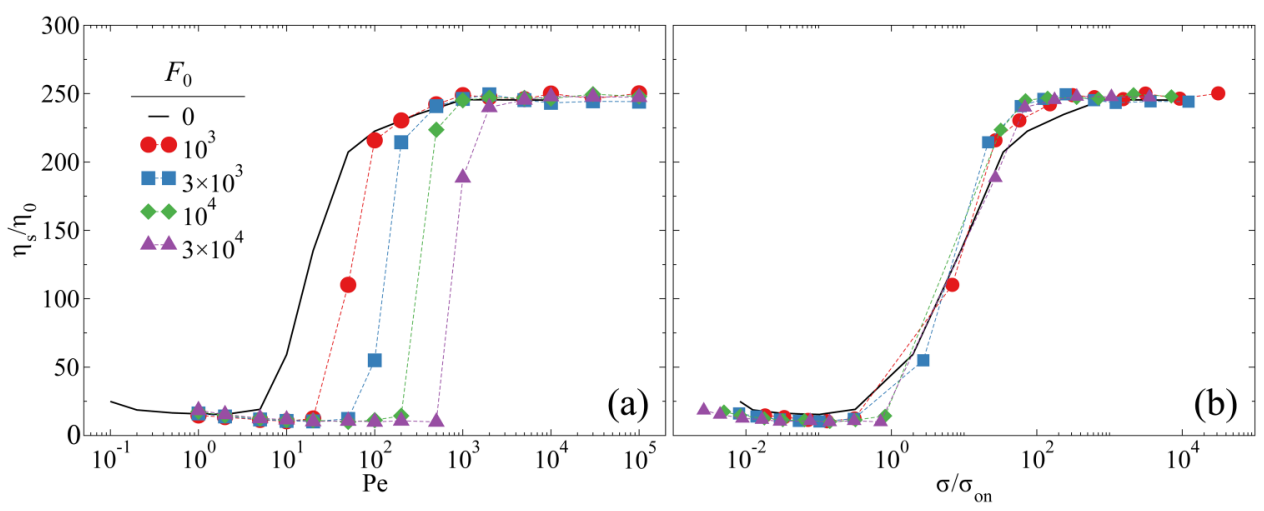

FIG. 6. The effects of a short-range repulsive force magnitude $F_{0}$ with $\tau a=100$ [see Eq. (1)] on the suspension shear viscosity $\eta_{s} / \eta_{0}$ for suspensions at $\phi=0.5, \alpha=0.01$, and $h_{0}=0.003$. (a) The shear viscosity as a function of Pe and (b) the shear viscosity as a function of the stress scaled with the onset stress, $\sigma / \sigma_{\text {on }}$. The onset stress $\sigma_{\text {on }} \sim F_{0} / a^{2}$, scaling as $a^{-2}$.

parameters in Table II reveal that $\alpha$ controls the magnitude of the shear thickening at high strain rates, and $F_{0}$ controls the onset strain rate for the shear thickening.

Figure 7 also shows the DPD simulation suspension viscosity at volume fractions $\phi=0.48,0.52$, and 0.56 as a function of Pe with $50 \%$ surface coverage and the asperity size of $0.05 a$. The Pe axis for the DPD results is on the top of the figure and is arranged to illustrate both quantitative and qualitative features comparing to the experimental and SEASD-nf data. Unlike SEASD-nf simulations, parameters in the DPD simulations are not tuned to match the experimental data. Nonetheless, with a shift of Pe, the suspension shear viscosity from DPD quantitatively agree with the experimental data. That the DPD simulations performed with a fixed surface coverage yield qualitative experimental agreement suggests that parameters $\alpha$ and $h_{0}$ in modified hydrodynamic interactions must change as a function of the volume fraction $\phi$ for given asperity descriptions in DPD, i.e., $\alpha=\alpha(\phi)$ and $h_{0}=h_{0}(\phi)$. We defer the exploration of such relations to future investigations.

Figures 8(a) and 8(b) compare the first and second normal stress differences, $N_{1}$ and $N_{2}$, respectively, between experiments and simulations. The normal stress differences $N_{1}$ and $\mathrm{N}_{2}$ are not used in the fitting process. With modified hydrodynamic interactions, we successfully captured the qualitative aspect of the experiments: both $N_{1}$ and $N_{2}$ are less than zero at high Pe. However, within the Pe range, the magnitude of the normal stress differences remains small compared to experimental observations. At lower Pe, normal stress differences from simulations are qualitatively consistent with studies on smooth particles. However, the experimental $N_{1}$

TABLE II. The fitted parameters for bidisperse coarse suspension simulations to match the experimental results of Cwalina and Wagner [12]. The coarse suspension is bidisperse with a size ratio $\lambda=1.4$ and a volume ratio $y_{s}=0.5$.

\begin{tabular}{lcllll}
\hline \hline Expt. & $\phi$ & $\alpha$ & $h_{0}$ & \multicolumn{1}{c}{$F_{0}$} & $\tau$ \\
\hline 1 & 0.50 & 0.013 & 0.0015 & $5 \times 10^{3}$ & 100 \\
2 & 0.54 & 0.04 & 0.0015 & $10^{4}$ & 100 \\
3 & 0.56 & 0.2 & 0.0015 & $2 \times 10^{4}$ & 100 \\
\hline \hline
\end{tabular}

and $N_{2}$ measurements at low strain rates are too noisy to facilitate meaningful comparisons.

The normal stress differences from the same DPD simulations in Fig. 7 are also presented in Fig. 8. Although the qualitative agreement between DPD and experiments remains, especially for the correct signs of $N_{1}$ and $N_{2}$, both $\mathrm{Pe}$ and stress scales are quite different, as is illustrated by the distinct scales in Fig. 8. The DPD normal stress differences are several orders of magnitude larger in the absolute value compared to experiments and SEASD-nf simulations. However, the DPD normal stress difference does asymptote to constant values in the high Pe limit, suggesting that they are material properties. This figure clearly illustrates both similarities and differences between the modified hydrodynamic interactions in SD and the explicit asperity description in DPD simulations for normal stress differences.

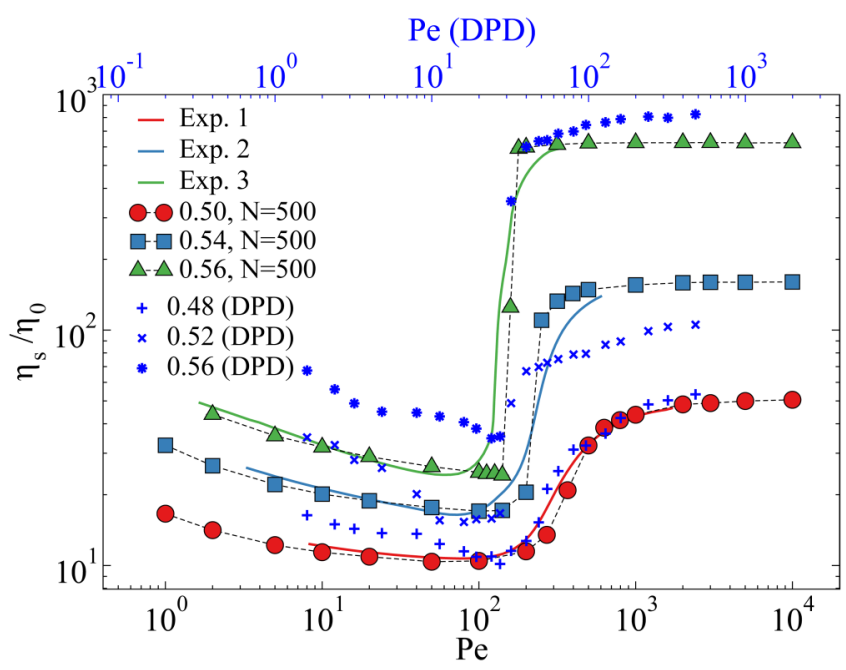

FIG. 7. The fitted suspension shear viscosity $\eta_{s} / \eta_{0}$ as a function of Pe. The solid lines represent experimental results of Cwalina and Wagner [12], and the symbols are the simulations from parameters in Table II. The suspension is bidisperse with size ratio $\lambda=1.4$ and volume ratio $y_{s}=0.5$. Also presented are the DPD simulation results at different volume fractions. Note that the Pe associated with DPD simulations are shown on the upper axis in blue. In DPD simulations, there no attempt was made to match the simulations with experiments. 

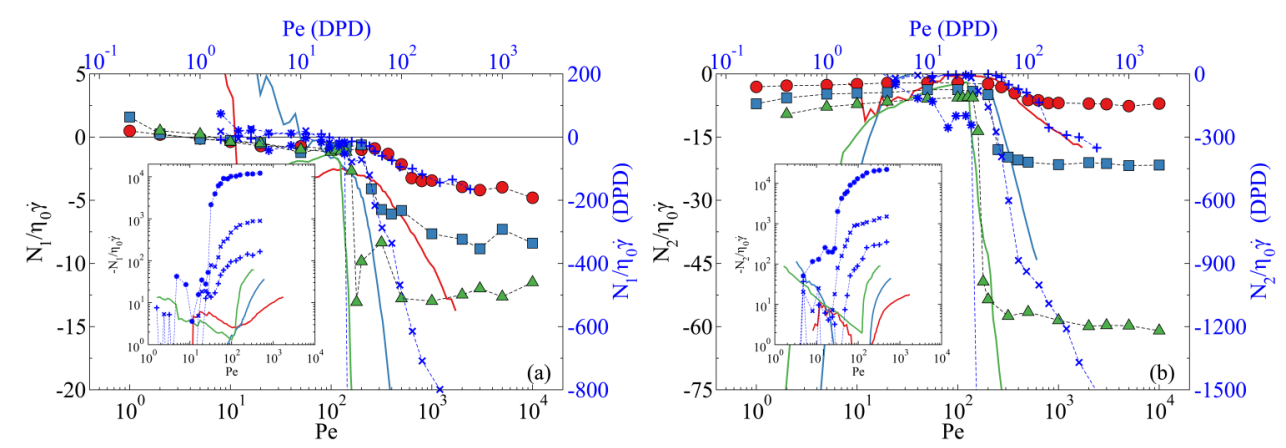

FIG. 8. The fitted suspension (a) first normal stress difference $N_{1} /\left(\eta_{0} \dot{\gamma}\right)$, (b) second normal stress difference $N_{2} /\left(\eta_{0} \dot{\gamma}\right)$ as functions of Péclet number $\mathrm{Pe}=\dot{\gamma} a_{s}^{2} / d_{0, s}$. The solid lines represent experimental results of Cwalina and Wagner [12], and the symbols are the simulations from parameters in Table II. Overlaid with the SD results are the DPD simulation results, where Pe and the normal stress differences are shown in blue. Both axes are shifted for the DPD simulations. The insets compare the DPD results with the experimental data using a logarithm stress scale. Symbols are identical to Fig. 7.

Having established a satisfactory agreement between experiments and simulations, we further examine the different components of the shear viscosity at $\phi=0.50$ and 0.56 in Figs. 9(a) and 9(b), respectively. Stokesian Dynamics allows us to identify different contributions of the suspension stress. We break down the suspension shear viscosity as $\eta_{s}=\eta_{0}+\eta_{B}+\eta_{E}+\eta_{P}$, where $\eta_{0}$ is the solvent viscosity, $\eta_{B}$ is the shear viscosity arising from the particle Brownian motion, $\eta_{E}$ is the viscosity contribution from the imposed flow, and $\eta_{P}$ is the viscosity due to the interparticle shortrange repulsive force. Figure 9 confirms the dominance of hydrodynamic interactions at high Pe. As expected, $\eta_{B}$ and $\eta_{P}$ reduce with increasing strain rates and exhibit qualitative changes near the onset of shear thickening. For instance, $\eta_{B} \sim \mathrm{Pe}^{-1}$ before the onset of shear thickening, but $\eta_{B} \sim$ $\mathrm{Pe}^{-2}$ afterward. These qualitative changes are especially pronounced in Fig. 9(b), where discontinuous jumps in $\eta_{B}, \eta_{E}$, and $\eta_{P}$ are observed with the onset of shear thickening.
These discontinuities indicate structural changes in suspension microstructure associated with the significant viscosity increase.

\section{Suspension structures}

The static structures of the sheared suspensions in real and wave spaces are examined in Figs. 10 and 11. Figure 10 shows a slice of the pair-distribution function $g(\mathbf{r})$ from the vorticity direction along the velocity-velocity gradient (12-) plane near the particle center at different volume fraction $\phi$ and Péclet number Pe. Due to the particle size bidispersity, we did not observe any crystallization in the suspensions. As Pe increases, the pair-distribution function $g(\mathbf{r})$ becomes increasingly distorted: particles accumulate on the compressional axis and are released along the extensional axis. Figure 11 shows the static structure factor $S(\mathbf{k})$ in the 12-plane with the vorticity direction wave vector $k_{3}=0$. The

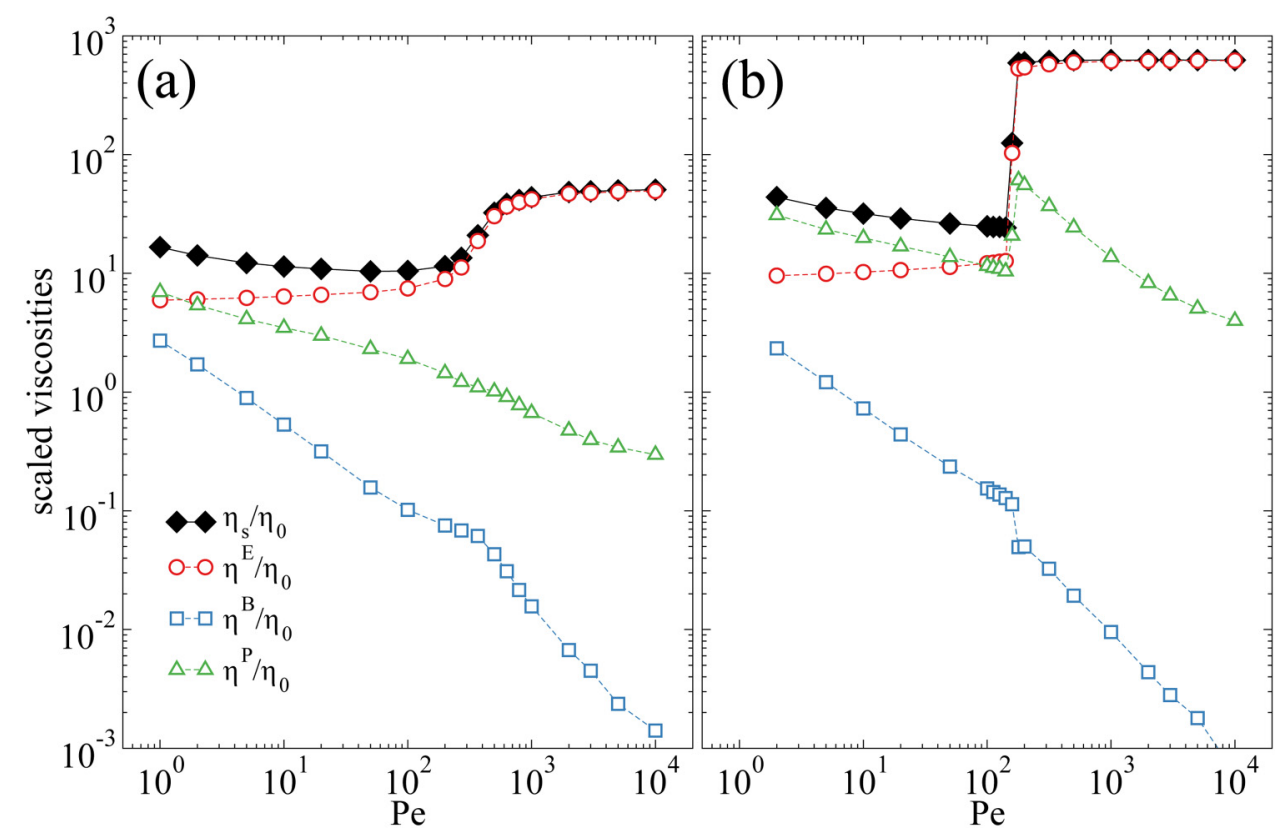

FIG. 9. Different contributions to the suspension shear viscosity for the fitted suspension at volume fractions (a) $\phi=0.50$ and (b) $\phi=0.56$ as functions of Pe. The suspension shear viscosity $\eta_{s}$ is shown in black diamonds. The flow viscosity contribution $\eta_{E}$ is shown in red circles, the Brownian contribution $\eta_{B}$ in blue squares, and the external force $\eta_{P}$ contribution is in green triangles. Simulation parameters are from Table II. 


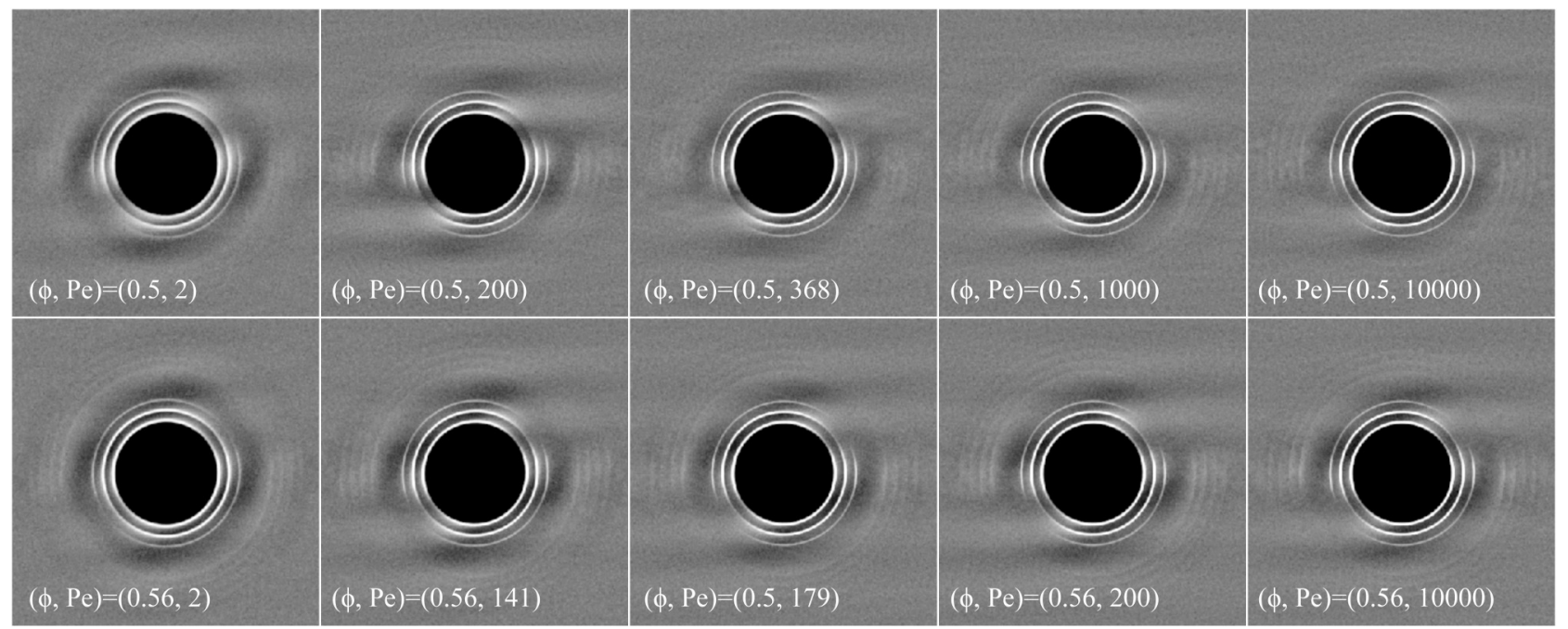

FIG. 10. The slice of pair-distribution function $g(\mathbf{r})$ in the shear (12-) plane cutting through the particle center with width $a_{s}$ in sheared suspensions at different volume fractions $\phi$ and Péclet number Pe with simulation parameters in Table II.

flow distortion to the suspension structures is similar to the $g(\mathbf{r})$ slices shown in Fig. 10 with a $90^{\circ}$-degree clockwise rotation.

These suspension structural features in Figs. 10 and 11 are consistent with earlier investigations of sheared bidisperse suspensions [41]. However, the viscosity jump associated with DST does not lead to significant structural changes in $g(\mathbf{r})$ and $S(\mathbf{k})$.

\section{Contact distribution in shear-thickened suspensions}

Figure 12 visualizes the suspension structures in our simulations at $\phi=0.5$ and 0.56 near the onset of discontinuous shear thickening in the velocity-velocity gradient (12-) plane. The particles are shown at $15 \%$ of their actual size for visibility and are colored based on their total shear stress. When two particles form a contact, i.e., when the gap spacing between two particles $h<h_{0}$, they are linked with a line. The instantaneous suspension shear viscosity within a few time units before and after the snapshot is also shown. Together with the suspension shear viscosity in Fig. 7, the number of contacts grows significantly after the onset of the viscosity jump for $(\phi, P e)=(0.5,1000)$, $(0.56,179)$, and $(0.56,10000)$. Also, the connections change qualitatively: before the onset of shear thickening, the stress connections are principally aligned with the compression axis of the simple shear flow. At higher strain rates, however, the connections are more likely to be aligned in the velocity and velocity gradient directions. These contact level features take place near the particle surfaces and are not easily captured by $g(\mathbf{r})$ in Fig. 10 and $S(\mathbf{k})$ in Fig. 11.

Although the suspension interactions are entirely hydrodynamic, the concept of contact helps us to understand how the short-range modified hydrodynamic interactions contribute to the suspension rheology. Consistent with Fig. 12, a contact is

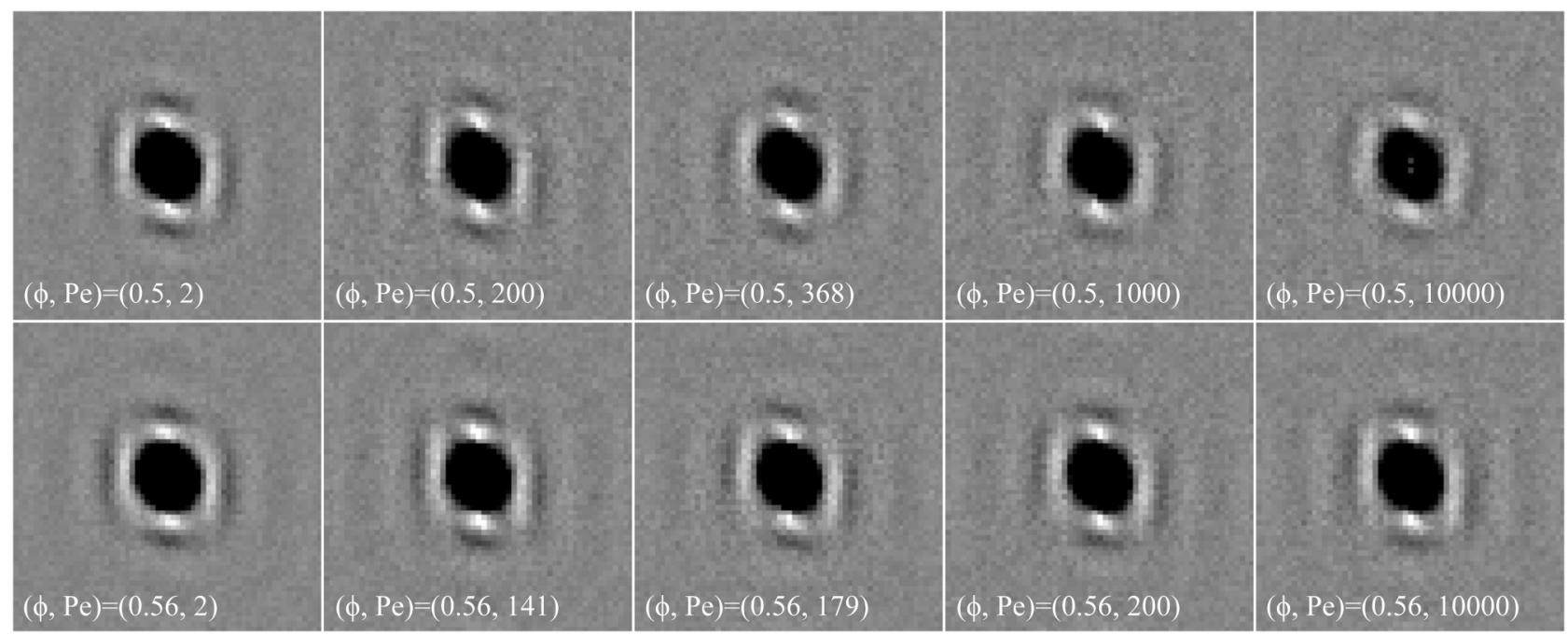

FIG. 11. The static structure factor $S(\mathbf{k})$ in the shear (12-) plane with a zero wave vector in the vorticity direction $k_{3}=0$ for sheared suspensions at different combinations of volume fraction $\phi$ and Péclet number Pe with simulation parameters in Table II. 
$(\phi, \mathrm{Pe})=(0.5,200)$

$(\phi, P e)=(0.5,368)$
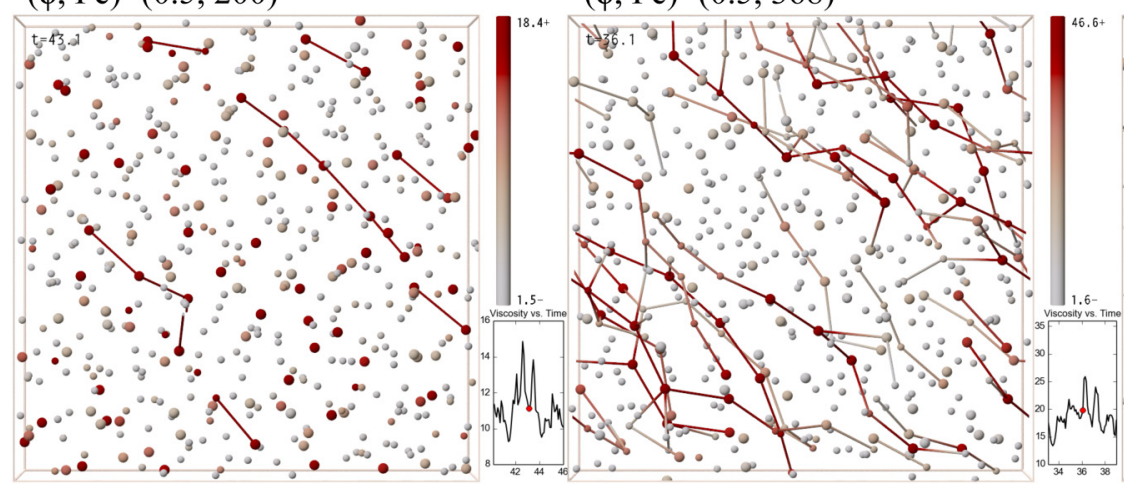

$(\phi, P e)=(0.5,1000)$

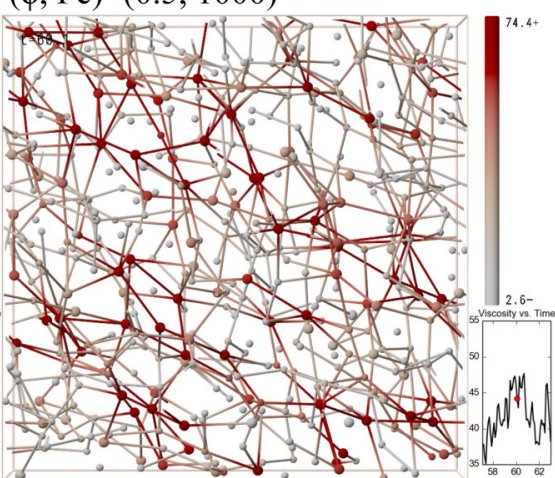

$(\phi, P e)=(0.56,141)$
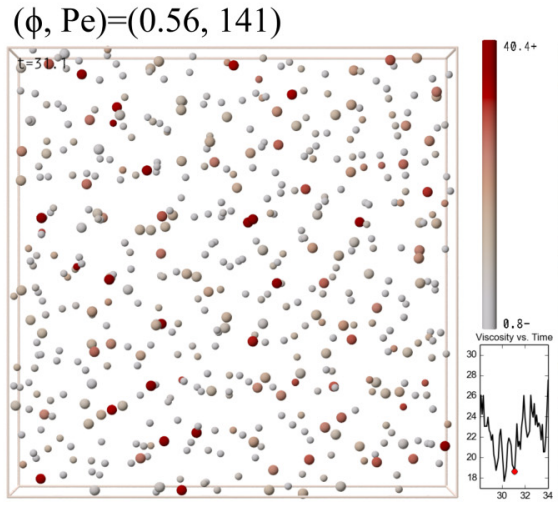

$(\phi, P e)=(0.56,10000)$

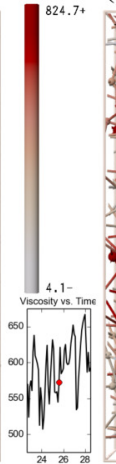

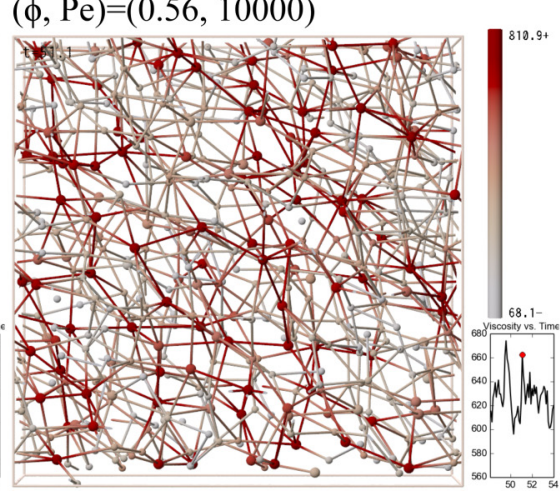

FIG. 12. Snapshots of suspensions in simulations at different volume fractions $\phi$ and Péclet number Pe. The particles are shown at $15 \%$ in actual size and are colored based on the magnitude of shear stress as indicated on the color bar. The particles are connected by a line when the pair forms a contact, i.e., when the gap spacing $h<h_{0}$ in Eq. (6). The small inset indicates instantaneous suspension shear viscosity $\eta_{s} / \eta_{0}$.

formed when a pair of particles are close to each other with the gap spacing $h \leq h_{0}$. Figure 13 illustrates the basic statistics of contacts in the suspensions as a function of Pe at different volume fractions $\phi$ based on the parameters of Table II. Figure 13(a) shows the average number of contacts per particle for particles having at least one contact, $Z_{\text {contact }}$, in the suspension. With Pe $<100$, the particles do not come close to each other and no contact could be identified in the suspension due to a low $\dot{\gamma}$ and a strong repulsive force. However, as the Péclet number grows beyond $\mathrm{Pe} \gtrsim 100$, $Z_{\text {contact }}$ emerges and grows rapidly with Pe. The number of contacts in the high shear limit increases with the volume fraction $\phi$. At $\phi=0.56$, in the high shear limit, $Z_{\text {contact }} \rightarrow 4$.2. At higher volume fractions $\phi$, the contacts
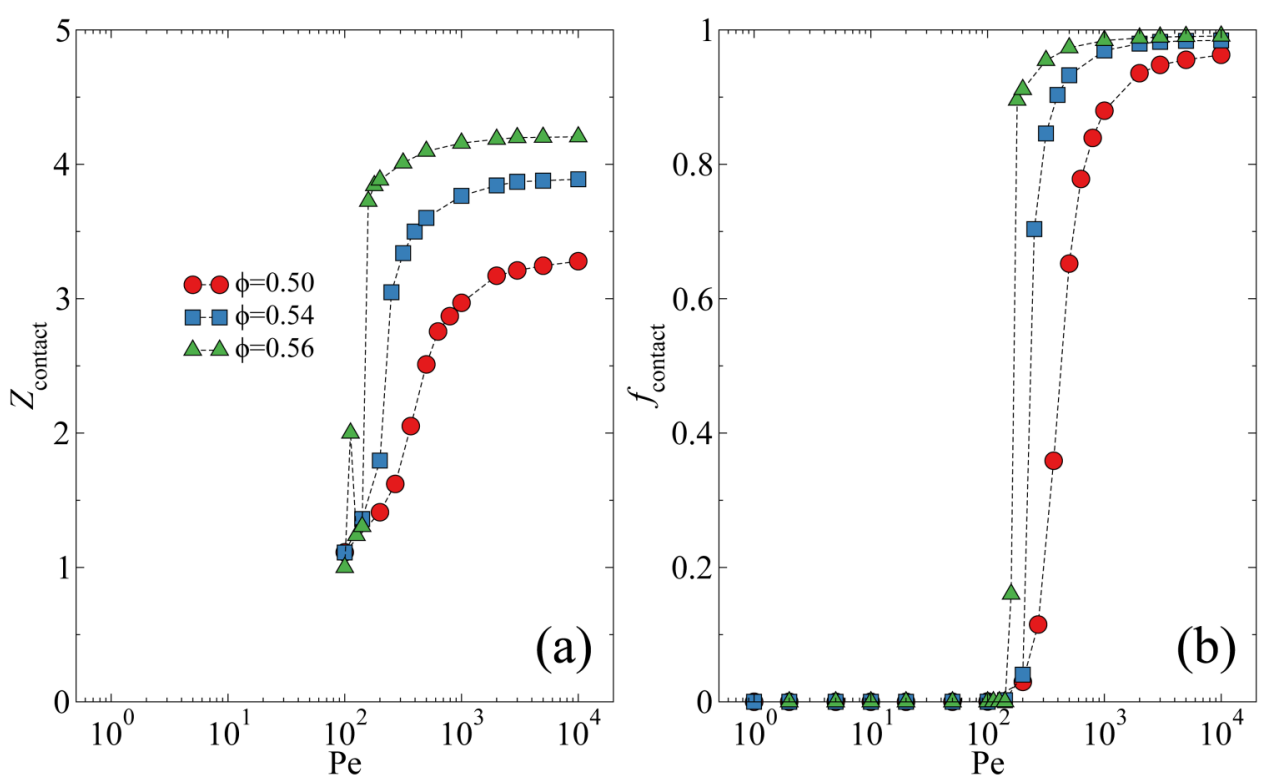

FIG. 13. The contact statistics of suspensions as a function of Péclet number Pe according to parameters in Table II. (a) The average number of contact $Z_{\text {contact }}$ per particle; (b) the fraction of particles containing at least one contact in the suspension, $f_{\text {contact }}$. Contacts are identified in the same way as in Fig. 12. 
increase more quickly with Pe near the shear thickening onset, suggesting faster activation of the modified hydrodynamic interactions.

Figure 13(b) displays the fractions of particles containing at least one contact, $f_{\text {contact }}$, in the suspensions. Similar to the observations in Fig. 13(a), $f_{\text {contact }}>0$ when $\mathrm{Pe} \gtrsim 100$. At higher Péclet numbers, the fraction of contact $f_{\text {contact }}$ grows quickly with Pe, suggesting the dominance of the modified hydrodynamic interactions. At the highest volume fraction $\phi=0.56, f_{\text {contact }} \rightarrow 1$ in the high shear limit, but at lower $\phi$, the maximum $f_{\text {contact }}$ does not reach 1 . Consistent with the observations in Fig. 13(a), $f_{\text {contact }}$ changes more rapidly with increasing Pe at higher $\phi$.

The contact distribution along the particle surface is shown in Fig. 14 at selected $\phi$ and Pe. To avoid doublecounting the contacts, we consider only the contact orientation within the northern hemisphere $(0<\varphi \leq \pi / 2)$ using a polar coordinate system commensurate with the simple shear flow, shown on the left of Fig. 14. The contact distribution is shown as a function of the cosine of the azimuthal angle $\mu=$ $\cos \varphi \in[0,1]$ and the polar angle $\theta \in[0,2 \pi)$. With $\mu=1$, the contact is at the "north pole" of the particle and with $\mu=0$ the contact is at the particle's equator.

Looking at the orientation distribution at $\phi=0.5$ and $\mathrm{Pe}=368$ in Fig. 14, the contacts are concentrated near $\theta \sim$ $\pi$ and $\mu \sim 1$. That the contacts are concentrated near $\theta \sim \pi$ implies that the contacts are concentrated along a slice in the shear plane intersecting the particle center. The distribution of $\mu$ suggests that the contacts are distributed almost uniformly from the compression axis $(\mu \approx 0.71)$ toward the "north pole" $(\mu=1)$. This is consistent with the observations of the pair-distribution functions in Fig. 10 and the suspension snapshot in Fig. 12.

The contact orientation distribution becomes more interesting for other $\phi$ and Pe combinations in Fig. 14, especially relative to $\mu$. Here, the orientation contacts become bimodal, with one close to $\mu \sim 1$ and another close to $\mu \sim 0$. The contacts are now concentrated in the front and the top of the particle. This is consistent with the more "cubical" appearance of the contact network in, for instance, the $(\phi, \mathrm{Pe})=(0.56,10000)$ case of Fig. 12. Physically, the contacts are formed as the particles

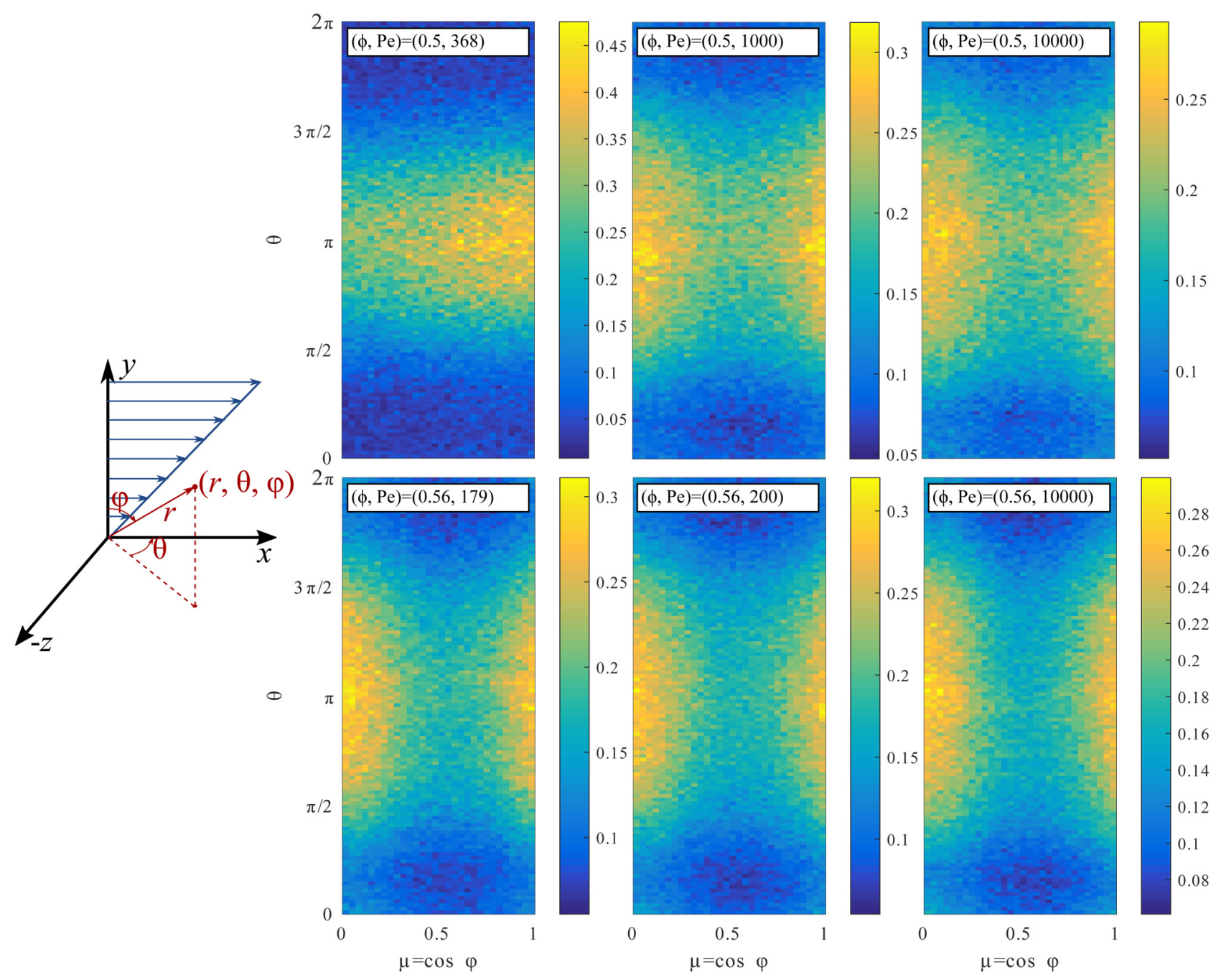

FIG. 14. Orientation distribution of particle contacts with respect to the polar angle $\theta$ and the cosine of the azimuthal angle $\varphi, \mu=\cos (\varphi)$, for suspensions at different combinations of volume fraction $\phi$ and Péclet number Pe. The orientation of the polar axis with respect to the simple shear flow is shown on the left. A contact is formed when the particle gap spacing $h<h_{0}$. The suspension simulation is based on parameters in Table II. 
approach each other "head on" at $\varphi=\pi / 2$, and the particle pair quickly rotates toward $\varphi=0$ and then disintegrates. During this cycle, the pair spends most of the time in its formation and disintegration stages. The distinct contact orientation distribution in Fig. 14 illustrates that the discontinuous shear thickening significantly changes the stress state of the suspension.

\section{Stress distribution and clustering in suspensions}

We further examine the stress distribution of the particles in Fig. 15 at selected combinations of $\phi$ and Pe consistent with Fig. 14. The red solid lines are the shear stress distribution of all particles in the suspension $P\left(\sigma_{12}\right)$, and the blue dashed lines are the stress distribution of particles with contacts $P_{c}\left(\sigma_{12}\right)$, normalized by the fraction of particles with at least one contact in the suspension $f_{\text {contact }}$, i.e., $f_{\text {contact }} \times P_{c}\left(\sigma_{12}\right)$.

At $\phi=0.50$ and $\mathrm{Pe}=368$, the suspension does not show strong discontinuous shear thickening: the stress in the suspension is dominated by unmodified hydrodynamic interactions. However, stresses arising from modified hydrodynamic lubrication contribute to a long tail with high-stress values and decay exponentially as $P\left(\sigma_{12}\right) \sim$ $\exp \left(-\sigma_{12}\right)$ as $\sigma_{12} \rightarrow \infty$. With increasing Pe at $\phi=0.5$, the hydrodynamic contact stress gradually dominates, and the stress contribution per particle diminishes.

At $\phi=0.56$, the stress probability distribution $P\left(\sigma_{12}\right)$ exhibits two distinct local maxima arising from unmodified and modified hydrodynamics. With increasing Pe, the peak per particle reduces but does not disappear. The two distinct peaks suggest that there are two populations of particles in the suspensions possessing distinct stress states.
To further probe the possibility of distinct stress states in the suspension, we study the spatial stress distribution of the suspension using a neighbor analysis. The analysis proceeds as follows: we first identify particles with the top five percentile stresses in the suspension, then we calculate the fraction of neighboring particles that also have the top five percentile of the suspension stress, $f_{n}$. A clustering metric is defined as $R=f_{n} / 0.05$ : that is, if $R>1$, the high-stress particles are more likely to appear together in clusters; on the other hand, if $R<1$, the high-stress particles are likely to avoid each other and they segregate. We identify the neighboring particles as the particle pairs sharing a neighboring cell in a radical tessellation [44].

The clustering metric $R$ as a function of Pe for three different suspensions investigated in this work is shown in Fig. 16. The clustering state before the onset of shear thickening is roughly identical, at $R \approx 1.8$. The parameter $R$ changes slowly with increasing Pe. Therefore, high-stress particles do appear together to a moderate degree in these suspensions. With the onset of shear thickening, $R$ increases significantly and then returns to an almost constant value lower than the value before the onset of shear thickening. Surprisingly, the high shear $R$ limiting value decreases with increasing volume fraction. Therefore, with the onset of shear thickening, the high shear stress particles still appear in clusters, but to a lesser extent compared to the low shear limit. At higher volume fractions, the stress distribution among particles is more spatially uniform.

Moreover, at the onset of discontinuous shear thickening, the suspension stress exhibits strong localization, as indicated by large peaks of $R$ values in Fig. 16. Therefore, the emergence of discontinuous shear thickening is a highly localized event.
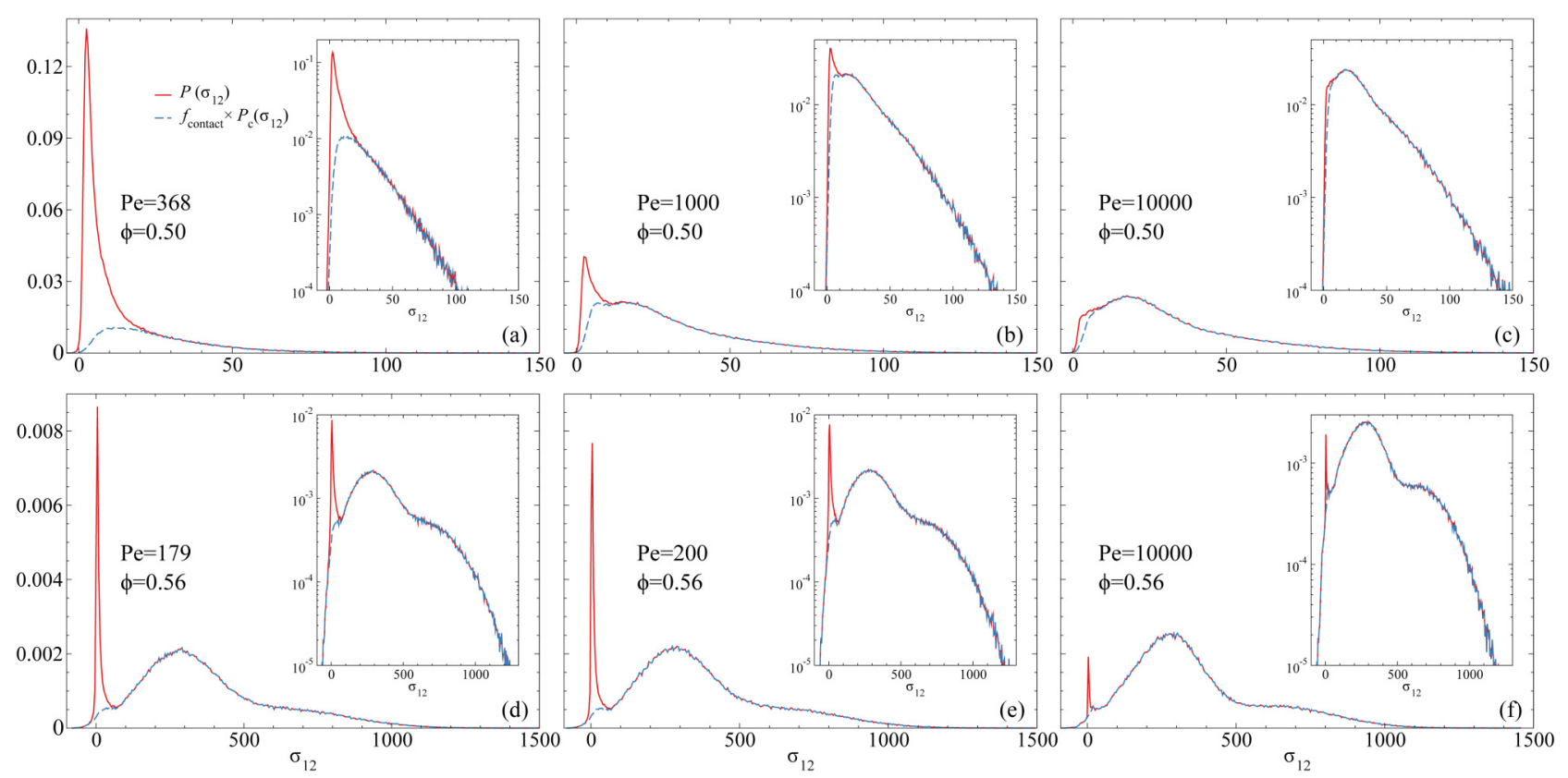

FIG. 15. Particle shear stress distribution at different volume fractions $\phi$ and Péclet numbers Pe for suspensions based on Table II. The red solid lines represent stress distributions of all particles in the suspension, and the blue dashed lines represent the contribution from particles with at least one contact, where a contact forms when the particle gap spacing $h<h_{0}$. 


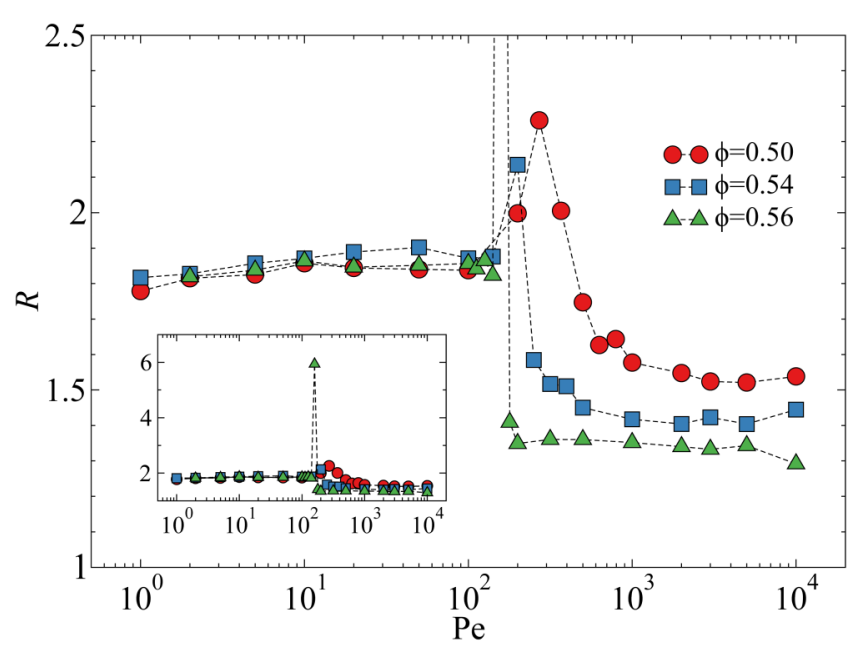

FIG. 16. The stress clustering metric, $R$, as functions of Péclet number Pe for suspensions in Table II. See text for the definition of the stress clustering metric $R$, where $R>1$ indicates that high-stress particles are likely to appear together, and $R<1$ indicates that high-stress particles avoid each other.

\section{Self-diffusion in shear-thickened suspensions}

Finally, we study particle self-diffusion in the vorticity direction as a function of the shear rate $\dot{\gamma}$ for suspensions in Fig. 17. The self-diffusion coefficient is evaluated from the slope of the particle mean-square displacement as a function of time, i.e., $d_{\infty}^{s}=\frac{1}{2} \mathrm{~d}\left\langle\Delta x_{3}^{2}\right\rangle / \mathrm{d} t$, where $\Delta x_{3}$ is the particle displacement in the vorticity direction where there is no mean drift. When scaled with the strain rate $\dot{\gamma} a^{2}$, the self-diffusion coefficient $d_{\infty}^{s}$ decreases with increasing Pe, and the magnitude of the strain rate scaled diffusion coefficient increases with increasing volume fractions. With the onset of discontinuous shear thickening, the diffusion coefficient $d_{\infty}^{s}$ approaches a constant value for different suspensions at different volume fractions, i.e., $d_{\infty}^{s} \approx 0.095 \dot{\gamma} a_{s}^{2}$. That the particle self-diffusion coefficients collapse at different volume fractions is consistent with the increased spatial stress uniformity in Fig. 16. Therefore, the high-stress state in DST introduces uniformity to the suspension microstructure.

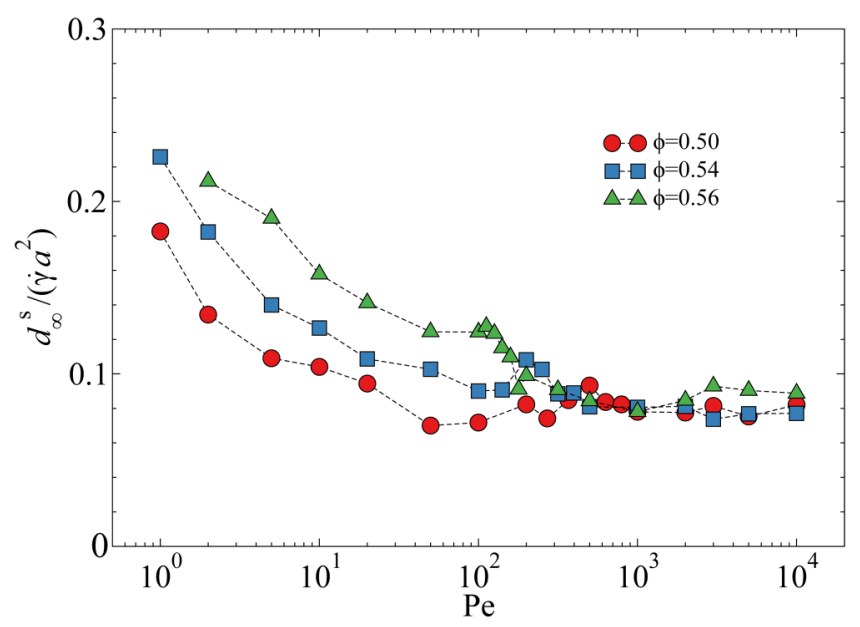

FIG. 17. The average long-time self-diffusivity in the vorticity direction, $d_{\infty}^{s} /\left(\dot{\gamma} a^{2}\right)$, as functions of Péclet number Pe for suspensions in Table II.

\section{CONCLUSIONS}

While constraints to sliding/rolling motion of colloidal particles are key components to recover the drastic changes in rheology of dense suspensions under flow, one cannot simply attribute such constraints to frictional contact between interacting particles. By fully resolving the effect of surface asperities in hydrodynamically interacting rough particles, we have demonstrated that lubrication forces and stresses borne in the fluid phase effectively result in hindered sliding motion and recover DST behavior in dense suspensions under flow. This can be done by taking into consideration the reduced gap between two surface asperities and the resulting tangential forces exerted on the colloid. Furthermore, one can account for such surface asperities by implicitly correcting the hydrodynamic lubrication (SD simulations presented) or by explicitly solving for spherical patches that represent surface roughness (DPD simulations presented and in [39]).

We have shown that the hydrodynamic stresses generated in reduced gaps between interacting rough particles result in DST behavior, where the rate dependence in the model is naturally introduced by the hydrodynamics. By decoupling the contributions to total bulk viscosities, we showed that the hydrodynamic viscosity dominates the stresses in the shearthickened state, in contrast to contact models in which the solid contact bears the majority of the total stress. Moreover, for corresponding repulsive forces, volume fractions, and the hydrodynamic ratio, the simulation results were fitted to experimental results of Cwalina and Wagner [12] without the need to tune/fit for simulation parameters for individual imposed deformation rates. Furthermore, both first and second normal stress differences in these suspensions remain negative and large, a hallmark of hydrodynamically interacting suspensions, in agreement with experimentally measured normal stress differences [12].

From a microstructural perspective, introducing surface asperities changes the number of neighbors (coordination number) of a particle in the shear-thickened state to $\sim 4$ for dense suspensions, similar to values observed for frictional particles [45]. Additionally, particle-particle bonds (referred to as colloids interacting through asperities) in the shear-thickened state are oriented in the velocity and velocity gradient directions, as opposed to compaction in the compressional axis traditionally observed in hydrodynamically interacting particles. Investigating the stress distribution in the system and the size of the particle cluster suggests that although stress is uniformly distributed in the system in the shear-thickened state, DST itself is a very localized event with a clear peak in stress cluster size at the onset of DST. From an experimental perspective, we believe that carefully designed transient rheology experiments, for instance, by combining flow reversal, stress jump, and highfrequency shear viscosity at orthogonal directions may help to elucidate the physical origin of DST [46,47].

\section{APPENDIX A: DPD SIMULATION WITH ROUGH PARTICLES}

The equation of motion for a DPD particle can be written as Eq. (A1), where each of the right-hand terms represents a 
pairwise interaction between two neighboring particles,

$$
m_{i} \frac{d \mathbf{v}_{i}}{d t}=\sum \mathbf{F}_{i j}^{C}+\mathbf{F}_{i j}^{D}+\mathbf{F}_{i j}^{R}+\mathbf{F}_{i j}^{H}+\mathbf{F}_{i j}^{\mathrm{Rep}} .
$$

The background Newtonian fluid is represented through explicit solvent particles that interact through the first three terms, and the colloidal particles are modeled through the last four terms in Eq. (A1). The conservative force, $\mathbf{F}_{i j}^{C}$ [Eq. (A2)], is exclusively used for the background fluid particles and represents the chemical identity and the compressibility of the fluid, based on GrootWarren expression [48],

$$
\mathbf{F}_{i j}^{C}=a_{i j}\left(1-\frac{r_{i j}}{r_{C}}\right) \mathbf{e}_{i j}
$$

The random force, $\mathbf{F}_{i j}^{R}$ [Eq. (A3)], represents the thermal fluctuations in the system and as a heat source for all particles through a random function, $\Theta_{i j}$ with a zero mean and a unit variance,

$$
\mathbf{F}_{i j}^{R}=\sigma_{i j}\left(1-\frac{r_{i j}}{r_{C}}\right) \frac{\Theta_{i j}}{\sqrt{\Delta t}} \mathbf{e}_{i j}
$$

The dissipative force, $\mathbf{F}_{i j}^{D}$ [Eq. (A4)], represents the viscous dissipation in the system and acts as the heat sink,

$$
\mathbf{F}_{i j}^{D}=-\gamma_{i j}\left(1-\frac{r_{i j}}{r_{C}}\right)^{2}\left(\mathbf{v}_{i j} \cdot \mathbf{e}_{i j}\right) \mathbf{e}_{i j}
$$

Thus, in this scheme, the random and the dissipative forces are coupled and should satisfy the fluctuationdissipation theorem [49] to provide the canonical ensemble required for proper representation of Brownian forces in the system. Hence, the dimensionless temperature in DPD is defined as $k T=\sigma_{i j}^{2} / 2 \gamma_{i j}$. In Eqs. (A2)-(A4), $r_{C}$ is the cutoff distance between two interacting particles that in DPD is equal to the diameter of the particle, $\mathbf{e}_{i j}$ is the unit vector $\mathbf{e}_{i j}=r_{i j}|| r_{i j} \mid, \mathbf{v}_{i j}=\mathbf{v}_{i}-\mathbf{v}_{j}$ is the relative velocity, $\sigma_{i j}$ is the strength of the thermal fluctuations, $\gamma_{i j}$ is the strength of dissipation, and $a_{i j}$ is the strength of conservative interactions between any $i$ th and $j$ th interaction particles.

The hydrodynamic force, $\mathbf{F}_{i j}^{H}$ [Eq. (A5)], represents the short-ranged lubrication forces based on the pair drag term, $\mu_{i j}^{H}=3 \pi \eta_{0} a_{i} a_{j} / 2 h_{i j}$, in the squeeze mode [20], which diverges at the surface-surface contact point, $h_{i j}=r_{i j}-a_{i}-a_{j}=0$. Thus, we regularize the hydrodynamic force at very small gaps of $\delta=10^{-4} a$,

$$
\mathbf{F}_{i j}^{H}=-\mu_{i j}^{H}\left(\mathbf{v}_{i j} \cdot \mathbf{e}_{i j}\right) \mathbf{e}_{i j} .
$$

The squeezing mode lubrication approximation is valid for small size ratios between two interacting particles, and thus, Eq. (A5) is only calculated for interacting base-base particles and for patch-patch particles [27,28,50]. The repulsive force, $\mathbf{F}_{i j}^{\text {Rep }}$ [Eq. (1)], represents the repulsive force existing between two interacting particles that naturally originates from the electrostatic double-layer, stabilizing charges, etc. and is a short-range interaction potential vanishing exponentially at $\tau a=100$ distance.

In DPD simulations presented here, a constant repulsion value of $F_{0}=10^{3} k_{\mathrm{B}} T / a$ is chosen. It should also be noted that the repulsive force is only calculated between the interacting base particles and not for the patch particles. Particle positions and velocities in each time step are advanced based on a velocity-Verlet algorithm [Eqs. (A6)-(A9)]. In this scheme, predictions of particle position [Eq. (A6)] and velocity [Eq. (A7)] are made based on the total force acting on a particle $\left[\mathbf{F}_{i}(t)\right]$, particle velocity $\left[\mathbf{v}_{i}(t)\right]$, and positions $\left[\mathbf{r}_{i}(t)\right]$ in the previous step,

$$
\begin{gathered}
\mathbf{r}_{i}(t+\Delta t)=\mathbf{r}_{i}(t)+\Delta t \mathbf{v}_{i}(t)+\frac{1}{2}(\Delta t)^{2} \mathbf{F}_{i}(t), \\
\tilde{\mathbf{v}}_{i}(t+\Delta t)=\mathbf{v}_{i}(t)+\frac{1}{2} \Delta t \mathbf{F}_{i}(t) .
\end{gathered}
$$

In the second step, new forces are calculated through Eqs. (1), (A2)-(A5), and corrections are made to the particle velocity [Eq. (A9)] based on this new total force [Eq. (A10)],

$$
\begin{gathered}
\mathbf{F}_{i}(t+\Delta t)=\mathbf{F}_{i}(\mathbf{r}(t+\Delta t), \tilde{\mathbf{v}}(t+\Delta t)), \\
\mathbf{v}_{i}(t+\Delta t)=\mathbf{v}_{i}(t)+\frac{1}{2} \Delta t\left(\mathbf{F}_{i}(t)+\mathbf{F}_{i}(t+\Delta t)\right) .
\end{gathered}
$$

Throughout the manuscript, quantities are expressed in nondimensional form. To do this, one should choose an energy scale, $k T$, a length scale that is commonly set by the cutoff distance, $r_{c}$, and the mass of individual particles, $m$, and derive the units for other quantities. Subsequently, one will have the following units: $(k T / m)^{1 / 2}$ for velocity, $r_{c}(m / k T)^{1 / 2}$ for time, $k T / r_{c}$ for force, $k T / m r_{c}$ for acceleration, $(k T / m)^{1 / 2} / r_{c}$ for shear rate, and $k_{\mathrm{B}} T / r_{c}^{3}$ for stress/pressure/ modulus. Further details of the simulation scheme, parametrization, and measurement of rheological properties can be found in previous reports [51-54]. The stress tensor is calculated using the Irving-Kirkwood [55,56] formalism, based on individual particle velocities, and the interactions acting in between pairs of particles and the total volume of the calculation box, $V$ [Eq. (A10)],

$\boldsymbol{\sigma}=\frac{1}{V}\left\{\sum_{i=1}^{N} m_{i}\left(\mathbf{v}_{i}-\mathbf{u}\left(r_{i}\right)\right) \otimes\left(\mathbf{v}_{i}-\mathbf{u}\left(r_{i}\right)\right)+\sum_{j>i}^{N} \sum_{i=1}^{N-1} \mathbf{r}_{i j} \otimes \mathbf{F}_{i j}\right\}$.

\section{ACKNOWLEDGMENTS}

M.W. was partially funded by NSERC PGS, and J.F.F. acknowledges support from NSF Grant No. CBET-1337097.

\section{REFERENCES}

[1] Brady, J. F., and G. Bossis, "The rheology of concentrated suspensions of spheres in simple shear flow by numerical simulation," J. Fluid Mech. 155, 105-129 (2006). 
[2] Mewis, J., and N. J. Wagner, Collodail Suspension Rheology. Cambridge Series in Chemical Engineering (Cambridge University, New York, 2012).

[3] Brown, E., and H. M. Jaeger, "Shear thickening in concentrated suspensions: Phenomenology, mechanisms and relations to jamming," Rep. Prog. Phys. 77(4), 046602 (2014).

[4] Morris, J. F., "A review of microstructure in concentrated suspensions and its implications for rheology and bulk flow," Rheol. Acta 48, 909-923 (2009).

[5] Barnes, H. A., "Shear-thickening (“dilatancy") in suspensions of nonaggregating solid particles dispersed in Newtonian liquids," J. Rheol. 33(2), 329-366 (1989).

[6] Hoffman, R. L., "Explanations for the cause of shear thickening in concentrated colloidal suspensions,” J. Rheol. 42(1), 111-123 (1998).

[7] Hoffman, R. L., "Discontinuous and dilatant viscosity behavior in concentrated suspensions. I. Observation of a flow instability," Trans. Soc. Rheol. 16(1), 155-173 (1972).

[8] Metzner, A. B., and M. Whitlock, "Flow behavior of concentrated (dilatant) suspensions," Trans. Soc. Rheol. 2(1), 239-254 (1958).

[9] Laun, H. M., "Normal stresses in extremely shear thickening polymer dispersions," J. Non-Newton. Fluid Mech. 54(0), 87-108 (1994).

[10] Aral, B. K., and D. M. Kalyon, "Viscoelastic material functions of noncolloidal suspensions with spherical particles," J. Rheol. 41(3), 599-620 (1997).

[11] Lee, M., M. Alcoutlabi, J. J. Magda, C. Dibble, M. J. Solomon, X. Shi, and G. B. McKenna, "The effect of the shear-thickening transition of model colloidal spheres on the sign of $\mathrm{N} 1$ and on the radial pressure profile in torsional shear flows," J. Rheol. 50(3), 293-311 (2006).

[12] Cwalina, C. D., and N. J. Wagner, "Material properties of the shearthickened state in concentrated near hard-sphere colloidal dispersions," J. Rheol. 58(4), 949-967 (2014).

[13] Hsiao, L. C., S. Jamali, E. Glynos, P. F. Green, R. G. Larson, and M. J. Solomon, "Rheological state diagrams for rough colloids in shear flow," Phys. Rev. Lett. 119(15), 158001 (2017).

[14] Madraki, Y., G. Ovarlez, and S. Hormozi, "Transition from continuous to discontinuous shear thickening: An excluded-volume effect," Phys. Rev. Lett. 121(10), 108001 (2018).

[15] Pan, Z., H. de Cagny, M. Habibi, and D. Bonn, "Normal stresses in shear thickening granular suspensions," Soft Matter 13(20), 3734-3740 (2017).

[16] Royer, J. R., D. L. Blair, and S. D. Hudson, "Rheological signature of frictional interactions in shear thickening suspensions," Phys. Rev. Lett. 116(18), 188301 (2016).

[17] Gamonpilas, C., J. F. Morris, and M. M. Denn, "Shear and normal stress measurements in non-Brownian monodisperse and bidisperse suspensions,” J. Rheol. 60(2), 289-296 (2016).

[18] Bossis, G., and J. F. Brady, "Dynamic simulation of sheared suspensions. I. General method," J. Chem. Phys. 80, 5141-5154 (1984).

[19] Bossis, G., and J. F. Brady, "The rheology of Brownian suspensions," J. Chem. Phys. 91, 1866-1874 (1989).

[20] Ball, R. C., and J. R. Melrose, "Lubrication breakdown in hydrodynamic simulations of concentrated colloids," Adv. Colloid Interface Sci. 59(0), 19-30 (1995).

[21] Melrose, J. R., and R. C. Ball, "The pathological behaviour of sheared hard spheres with hydrodynamic interactions," Europhys. Lett. 32(6), 535-540 (1995).

[22] Silbert, L. E., J. R. Melrose, and R. C. Ball, "Colloidal microdynamics: Pair-drag simulations of model-concentrated aggregated systems," Phys. Rev. E 56(6), 7067 (1997).

[23] Foss, D. R., and J. F. Brady, "Structure, diffusion and rheology of Brownian suspensions by Stokesian dynamics simulation," J. Fluid Mech. 407, 167-200 (2000).
[24] D'Haene, P., J. Mewis, and G. G. Fuller, "Scattering dichroism measurements of flow-induced structure of a shear thickening suspension," J. Colloid Interface Sci. 156(2), 350-358 (1993).

[25] Bender, J., and N. J. Wagner, "Reversible shear thickening in monodisperse and bidisperse colloidal dispersions," J. Rheol. 40(5), 899-916 (1996).

[26] Melrose, J. R., and R. C. Ball, "Continuous shear thickening transitions in model concentrated colloids-The role of interparticle forces," J. Rheol. 48(5), 937-960 (2004).

[27] Jamali, S., M. Yamanoi, and J. Maia, "Bridging the gap between microstructure and macroscopic behavior of monodisperse and bimodal colloidal suspensions," Soft Matter 9(5), 1506-1515 (2013).

[28] Jamali, S., and A. Boromand, "Microstructure and rheology of soft to rigid shear-thickening colloidal suspensions," J. Rheol. 59(6), 1377-1395 (2015).

[29] Kim, S., and S. J. Karrila, Microhydrodynamics Principles and Selected Applications (Dover, Mineola, New York, 1991).

[30] Mari, R., R. Seto, J. F. Morris, and M. M. Denn, "Shear thickening, frictionless and frictional rheologies in non-Brownian suspensions," J. Rheol. 58(6), 1693-1724 (2014).

[31] Mari, R., R. Seto, J. F. Morris, and M. M. Denn, "Discontinuous shear thickening in Brownian suspensions by dynamic simulation," Proc. Natl. Acad. Sci. U.S.A. 112(50), 15326 (2015).

[32] Morris, J. F., "Lubricated-to-frictional shear thickening scenario in dense suspensions," Phys. Rev. Fluids 3(11), 110508 (2018).

[33] Seto, R., R. Mari, J. F. Morris, and M. M. Denn, "Discontinuous shear thickening of frictional hard-sphere suspensions," Phys. Rev. Lett. 111(21), 218301 (2013).

[34] Wyart, M., and M. E. Cates, "Discontinuous shear thickening without inertia in dense non-Brownian suspensions," Phys. Rev. Lett. 112(9), 098302 (2014).

[35] Fernandez, N., R. Mani, D. Rinaldi, D. Kadau, M. Mosquet, H. Lombois-Burger, J. Cayer-Barrioz, H. J. Herrmann, N. D. Spencer, and L. Isa, "Microscopic mechanism for shear thickening of non-Brownian suspensions," Phys. Rev. Lett. 111(10), 108301 (2013).

[36] Hsu, C.-P., S. N. Ramakrishna, M. Zanini, N. D. Spencer, and L. Isa, "Roughness-dependent tribology effects on discontinuous shear thickening," Proc. Natl. Acad. Sci. U.S.A. 115(20), 5117-5122 (2018)

[37] Schroyen, B., B. Schroyen, C.-P. Hsu, L. Isa, P. V. Puyvelde, and J. Vermant, "Stress contributions in colloidal suspensions: The smooth, the rough, and the hairy," Phys. Rev. Lett. 122(21), 218001 (2019).

[38] Hsiao, L. C., I. Saha-Dalal, R. G. Larson and M. J. Solomon, "Translational and rotational dynamics in dense suspensions of smooth and rough colloids," Soft Matter 13(48), 9229-9236 (2017).

[39] Jamali, S., and J. F. Brady, "Alternative frictional model for discontinuous shear thickening of dense suspensions: Hydrodynamics," Phys. Rev. Lett. 123(13), 138002 (2019).

[40] Brady, J. F., and G. Bossis, "Stokesian dynamics," Annu. Rev. Fluid Mech. 20(1), 111-157 (1988).

[41] Wang, M., and J. F. Brady, "Short-time transport properties of bidisperse suspensions and porous media: A Stokesian dynamics study," J. Chem. Phys. 142(9), 094901 (2015).

[42] Swan, J. W., and J. F. Brady, "The hydrodynamics of confined dispersions," J. Fluid Mech. 687, 254-299 (2011).

[43] Wang, M., and J. F. Brady, "Spectral Ewald acceleration of Stokesian dynamics for polydisperse suspensions," J. Comput. Phys. 306, 443-477 (2016).

[44] Rycroft, C. H., "Voro++: A three-dimensional Voronoi cell library in C++," Chaos 19(4), 041111 (2009). 
[45] Guy, B. M., J. A. Richards, D. J. M. Hodgson, E. Blanco, and W. C. K. Poon, "Constraint-based approach to granular dispersion rheology," Phys. Rev. Lett. 121(12), 128001 (2018).

[46] Lin, N. Y. C., B. M. Guy, M. Hermes, C. Ness, J. Sun, W. C. K. Poon, and I. Cohen, "Hydrodynamic and contact contributions to continuous shear thickening of colloids suspension," Phys. Rev. Lett. 115, 228304 (2015).

[47] Lin, N. Y. C., C. Ness, M. E. Cates, J. Sun, and I. Cohen, "Tunable shear thickening in suspensions," PNAS 113(39), 10774-10778 (2016).

[48] Groot, R. D., and P. B. Warren, "Dissipative particle dynamics: Bridging the gap between atomistic and mesoscopic simulation," J. Chem. Phys. 107(11), 4423-4435 (1997).

[49] Espanol, P., and P. Warren, "Statistical mechanics of dissipative particle dynamics," Europhys. Lett. 30(4), 191-196 (1995).

[50] Boromand, A., S. Jamali, B. Grove, and J. M. Maia, "A generalized frictional and hydrodynamic model of the dynamics and structure of dense colloidal suspensions," J. Rheol. 62(4), 905-918 (2018).
[51] Boromand, A., S. Jamali, and J. M. Maia, "Viscosity measurement techniques in dissipative particle dynamics," Comput. Phys. Commun. 196, 149-160 (2015).

[52] Boromand, A., S. Jamali, and J. M. Maia, "Structural fingerprints of yielding mechanisms in attractive colloidal gels," Soft Matter 13(2), 458-473 (2017).

[53] Jamali, S., A. Boromand, S. Khani, and J. Maia, "Gaussian-inspired auxiliary non-equilibrium thermostat (GIANT) for dissipative particle dynamics simulations," Comput. Phys. Commun. 197, 27-34 (2015).

[54] Jamali, S., G. H. McKinley, and R. C. Armstrong, "Microstructural rearrangements and their rheological implications in a model thixotropic elastoviscoplastic fluid,” Phys. Rev. Lett. 118(4), 048003 (2017).

[55] Irving, J. H., and J. G. Kirkwood, "The statistical mechanical theory of transport processes. IV. The equations of hydrodynamics," J. Chem. Phys. 18(6), 817-829 (1950).

[56] Kirkwood, J. G., and F. P. Buff, "The statistical mechanical theory of surface tension,” J. Chem. Phys. 17(3), 338-343 (1949). 